\title{
FIXED POINTS AND AMENABILITY IN NON-POSITIVE CURVATURE
}

\author{
PIERRE-EMMANUEL CAPRACE* AND NICOLAS MONOD ${ }^{\ddagger}$
}

\begin{abstract}
Consider a proper cocompact CAT(0) space $X$. We give a complete algebraic characterisation of amenable groups of isometries of $X$. For amenable discrete subgroups, an even narrower description is derived, implying Q-linearity in the torsionfree case.

We establish Levi decompositions for stabilisers of points at infinity of $X$, generalising the case of linear algebraic groups to $\operatorname{Is}(X)$. A geometric counterpart of this sheds light on the refined bordification of $X$ (à la Karpelevich) and leads to a converse to the Adams-Ballmann theorem. It is further deduced that unimodular cocompact groups cannot fix any point at infinity except in the Euclidean factor; this fact is needed for the study of $\mathrm{CAT}(0)$ lattices.

Various fixed point results are derived as illustrations.
\end{abstract}

\section{Contents}

1. Introduction 1

2. On the structure of locally compact groups 6

3. Horoactions and Levi decompositions 9

4. Compactible subgroups and unimodularity 18

5. Amenable isometry groups 20

References 31

\section{INTRODUCTION}

1.A. Amenable isometry groups. A celebrated theorem by Tits asserts that an arbitrary group $G \subseteq \mathrm{GL}(V)$ of linear transformations of a finite-dimensional vector space $V$ over any field is subjected to the following alternative: either $G$ contains a non-abelian free subgroup, or $G$ is soluble-by-\{locally finite\} (see Theorems 1 and 2 in [Tit72]). In particular a subgroup $G \subseteq \mathrm{GL}(V)$ is amenable if and only if it is soluble-by-\{locally finite $\}$. The importance of that result stimulated since then an active search for larger classes of groups satisfying a similar alternative. It is in particular a notorious open problem to obtain a version of the Tits alternative for groups $G \subseteq \operatorname{Is}(X)$, where $X$ is a cocompact proper CAT(0) space, i.e. a non-positively curved proper metric space with a cocompact isometry group.

Date: March 2012.

* F.R.S.-FNRS research associate. Supported in part by FNRS grant F.4520.11 and by the ERC.

${ }^{\ddagger}$ Supported in part by the Swiss National Science Foundation and the ERC. 
While considering extensions of the Tits alternative to wider families of groups, it is natural to split the problem into two sub-questions, namely:

(1) Does every non-amenable subgroup contain a free group?

(2) What is the algebraic structure of amenable subgroups?

Our first goal in this paper is to provide a complete answer to Question (2) for isometry groups of a cocompact proper CAT(0) space. To this end, we recall that the locally elliptic radical $\operatorname{Rad} \mathscr{L} \mathscr{E}$ of a locally compact group is the largest normal subgroup which can be written as increasing union of compact groups (see [Pla65]). In case of discrete groups, locally elliptic is thus a synonym of locally finite.

The following theorem shows that a subgroup is amenable if and only if it has a specific canonical decomposition into pieces that are either connected soluble or discrete soluble or locally elliptic, in analogy with Tits' description of amenable linear groups.

Theorem A. Let $X$ be a proper cocompact $\mathrm{CAT}(0)$ space. hold:

A closed subgroup $H \subseteq \operatorname{Is}(X)$ is amenable if and only if the following three conditions

(1) $H^{\circ}$ is soluble-by-compact,

(2) $H^{\circ} \operatorname{Rad}_{\mathscr{L} \mathscr{E}}(H)$ is open in $H$,

(3) $H /\left(H^{\circ} \operatorname{Rad} \mathscr{L} \mathscr{E}(H)\right)$ is virtually soluble.

Thus a closed subgroup $H \subseteq \operatorname{Is}(X)$ is amenable if and only if it is \{connected soluble\}by-\{locally elliptic\}-by-\{discrete virtually soluble\}.

In the same way as the Tits alternative can be used to find obstructions to linearity, Theorem A implies that many groups cannot appear as closed subgroups of a cocompact group of isometries of a proper CAT(0) spaces. Since amenability passes to the closure, we still get restrictions on arbitrary amenable subgroups. As an extreme example, we recall that there is an active search for (infinite) finitely generated simple amenable groups; non-positively curved spaces will not be the natural habitat where to hunt for them:

Corollary B. Let $\Gamma$ be an infinite finitely generated amenable group. Assume that the only virtually abelian quotient of $\Gamma$ is the trivial one (e.g. $\Gamma$ is simple).

Then there is no non-trivial isometric $\Gamma$-action whatsoever on any proper cocompact $\mathrm{CAT}(0)$ space.

1.B. Discrete amenable subgroups. Given a discrete group $\Gamma$ acting properly and cocompactly on $X$, all the amenable subgroups of $\Gamma$ are virtually abelian and stabilise a flat in $X$ : this was proved by Adams-Ballmann (Corollary B in [AB98]) and generalises the Solvable Subgroup Theorem (Theorem II.7.8 in [BH99]).

We emphasize that Theorem A does not suppose the existence of any discrete cocompact group of isometries: only the (possibly indiscrete) full isometry group $\operatorname{Is}(X)$ is assumed cocompact. Although discrete amenable subgroups of $\operatorname{Is}(X)$ need not be virtually abelian (for instance the Heisenberg group over $\mathbf{Z}$ is a discrete subgroup of $\mathrm{SL}_{3}(\mathbf{R})$ ), Theorem A states that they must be $\{$ locally finite $\}$-by-\{virtually soluble\}. We remark that the virtually soluble quotient need not be finitely generated in general (this applies a fortiori to the discrete quotient of $H$ in Theorem A). Indeed, a CAT(0) lattice such as $\mathrm{SL}_{n}(\mathbf{Z}[1 / p])$ contains the infinitely generated abelian group $\mathbf{Z}[1 / p]$ as a subgroup. 
Notice however that every finitely generated subgroup of $\mathbf{Z}[1 / p]$ is cyclic. This reflects a general property of $\mathbf{Q}$-linear soluble groups, all of which have finite Prüfer rank. Recall that this rank is the smallest integer $r$ such that every finitely generated subgroup can be generated by at most $r$ elements. Our next theorem establishes such a finiteness result in the generality of all proper cocompact $\mathrm{CAT}(0)$ spaces.

Theorem C. Let $X$ be a proper cocompact $\mathrm{CAT}(0)$ space. Then there is a constant $r=r(X)$ such that the following holds.

For every discrete amenable subgroup $\Gamma<\operatorname{Is}(X)$, the quotient $\Gamma / \operatorname{Rad} \mathscr{L L E}_{\mathscr{E}}(\Gamma)$ is virtually $\{$ torsion-free soluble of Prüfer rank $\leq r\}$.

Remark that for each prime $p$, the lamplighter group $(\mathbf{Z} / p) \imath \mathbf{Z}$ can be realised as a discrete group of isometries of a cocompact CAT(0) space, since it embeds in the CAT(0) lattice $\mathrm{SL}_{n}\left(\mathbf{F}_{p}\left[t, t^{-1}\right]\right)$. Theorem $\mathrm{C}$ implies that, on the other hand, the wreath product $\mathbf{Z}$ ? $\mathbf{Z}$ cannot.

Since torsion-free soluble groups of finite Prüfer rank are known to be linear over $\mathbf{Q}$ by a theorem of Wehrfritz [Weh73, pp.25-26], Theorem C has the following consequence.

Corollary D. Let $X$ be a proper cocompact $\mathrm{CAT}(0)$ space.

Then any torsion-free discrete amenable subgroup $\Gamma<\operatorname{Is}(X)$ is $\mathbf{Q}$-linear.

By a theorem of Sh. Rosset [Ros76], the kernel of any homomorphism of a finitely generated group of subexponential growth to a virtually soluble group is itself finitely generated. As pointed out to us by Ami Eisenmann, the latter fact combined with Theorem C yields the following, since a virtually soluble group of subexponential growth is virtually nilpotent [Mil68].

Corollary E. Let $X$ be a proper cocompact CAT(0) space.

Then every finitely generated discrete subgroup $\Gamma<\operatorname{Is}(X)$ of subexponential growth is virtually nilpotent.

Thus $\operatorname{Is}(X)$ does not admit discrete subgroups of intermediate growth.

Of course certain groups of intermediate growth can be embedded non-discretely into Is $(X)$, for instance if they are residually finite like Grigorchuk's group.

1.C. Refining spaces. A fundamental tool for the study of amenable subgroups of isometries of a proper CAT(0) space $X$ is the Adams-Ballmann theorem [AB98] which states that such a group preserves a flat or fixes a point at infinity. The first case of this alternative is of course highly satisfactory since the isometries of Euclidean space form a very elementary Lie group, but the case of a fixed point at infinity seems at first sight to be of little help for the elucidation of the group.

The way forward here is to use the transverse space $X_{\xi}$ of a point $\xi \in \partial X$ together with the canonical action of the stabiliser of $\xi$ on $X_{\xi}$, see Section 3.D below (in the classical case of symmetric spaces, this construction goes back at least to the Karpelevich compactification; in general, see also [Lee00], [Cap09]). This opens the door to iterations, considering refining sequences $\left(\xi_{1}, \ldots, \xi_{k}\right)$, which are defined by $\xi_{1} \in \partial X$ and then $\xi_{i+1} \in \partial X_{\xi_{1}, \ldots, \xi_{i}}$. A refined point, flat, etc. refers to the corresponding object in some $X_{\xi_{1}, \ldots, \xi_{k}}$. With this terminology at hand, we can state a converse to the Adams-Ballmann theorem, whose proof relies on iterative constructions of liftings for transverse spaces with 
(generally non-continuous) associated homomorphisms, and on an appropriate version of the Kazhdan-Margulis theorem.

Theorem F. Let $X$ be a proper cocompact CAT(0) space.

Then the stabiliser of every refined flat of $X$ is amenable (and closed).

This is a converse because both theorems combine to give the following geometric characterisation of all amenable subgroups, thus complementing the algebraic characterisation of Theorem A:

Corollary G. Let $X$ be a proper cocompact CAT(0) space.

Then a closed subgroup of $\operatorname{Is}(X)$ is amenable if and only if it preserves a refined flat.

The 'only if' direction follows from a simple iteration of the Adams-Ballmann theorem [AB98]. This iteration process terminates after finitely many steps because the maximal index $k$ of a refining sequence $\left(\xi_{1}, \ldots, \xi_{k}\right)$ in $X$ is bounded when $X$ is cocompact. This bound, which we call the depth of $X$, turns out to coincide with the flat rank (see Corollary 3.20 below).

There are groups for which every isometric action on a proper $\operatorname{CAT}(0)$ space must preserve a refined flat without requesting the amenability of the group. It is easy to produce such examples in a way that runs afoul of the combination of Corollary G and Theorem A, thus giving groups that cannot act at all. Here is an example:

Corollary H. Richard Thompson's simple groups $T$ and $V$ do not admit any non-trivial isometric action whatsoever on any proper cocompact $\mathrm{CAT}(0)$ space.

Recall that Thompson's group $T$ can be viewed as the group of all orientation preserving piecewise affine transformations of $\mathbf{R} / \mathbf{Z}$ which have dyadic breaking points and whose slopes are integral powers of two. Alternatively, it admits the following finite presentation, see [CFP96, §5].

$$
\begin{aligned}
T=\langle a, b, c| & {\left[a b^{-1}, a^{-1} b a\right],\left[a b^{-1}, a^{-2} b a^{2}\right], c^{-1} b a^{-1} c b, } \\
& \left.\left(a^{-1} c b a^{-1} b a\right)^{-1} b a^{-2} c b^{2}, a^{-1} c^{-1}\left(a^{-1} c b\right)^{2}, c^{3}\right\rangle
\end{aligned}
$$

This group is known to have a very nice (proper) isometric action on a locally finite CAT(0) cube complex [Far05], but the latter space is not cocompact - indeed not finitedimensional. A similar construction is available for $V$.

At the end of Section 5.B, we shall give various examples of groups, amenable or not, some of them torsion-free, for which no isometric action on any proper cocompact CAT(0) space can be faithful. Recall that every countable group embeds in a 2-generated simple group (see [Hal74] and [Sch76]). Moreover, if the countable group in question is torsion-free, then the simple group can also be chosen torsion-free, as follows from Theorem A in [Mei85] (see also Remark 2 on p. 392 in loc.cit.). Applying this embedding theorem to one of the torsion-free groups without faithful action on a proper cocompact CAT(0) space constructed in Section 5.B below, we obtain the following.

Corollary I. There is an infinite 2-generated torsion-free simple group that does not admit any non-trivial isometric action on any proper cocompact $\mathrm{CAT}(0)$ space. 
Notice that the assumption that a space be cocompact, which does not impose any restriction on the action of the groups that we consider, does not either imply any sort of local regularity nor of finite dimensionality. For very explicit examples of proper cocompact minimal CAT $(-1)$ spaces that are infinite-dimensional, see [MP12].

1.D. Fixed points and Levi decompositions. In the quest for understanding cocompact CAT(0) spaces and their cocompact isometry groups, we have proposed in [CM09b] some structural results under the assumption that no point at infinity be fixed by the entire cocompact group. Notice however that the results stated above do not request that hypothesis; their proofs therefore require to study the remaining case, i.e when some point at infinity has a stabiliser acting cocompactly on the space. We shall prove that such a stabiliser admits a Levi decomposition generalising the situation of parabolic subgroups of semi-simple algebraic groups; moreover, the corresponding "Levi factor" will again be represented as a cocompact isometry group of a suitable CAT(0) subspace.

Let thus $X$ be a proper $\mathrm{CAT}(0)$ space, $G<\mathrm{Is}(X)$ a closed subgroup and $\xi \in \partial X$. We define

$$
G_{\xi}^{\mathrm{u}}:=\left\{g \in G: \lim _{t \rightarrow \infty} d(g \cdot r(t), r(t))=0 \quad \forall r \text { with } r(\infty)=\xi\right\}
$$

(where $r: \mathbf{R} \rightarrow X$ are geodesic rays). This is a closed normal subgroup of $G_{\xi}$. We recall that $\operatorname{Opp}(\xi)$ is the set of points $\xi^{\prime} \in \partial X$ that are visually opposite $\xi$, i.e. such that there is a bi-infinite geodesic line in $X$ with extremities $\xi$ and $\xi^{\prime}$. The union of all such lines is denoted by $P\left(\xi, \xi^{\prime}\right)$; it is a closed convex subspace of $X$ with a non-trivial Euclidean factor. If the stabiliser $\operatorname{Is}(X)_{\xi}$ acts cocompactly, then the set $\operatorname{Opp}(\xi)$ is non-empty.

Theorem J. Assume that $G_{\xi}$ acts cocompactly on $X$. Then for each $\xi^{\prime} \in \operatorname{Opp}(\xi)$ we have a decomposition

$$
G_{\xi}=G_{\xi, \xi^{\prime}} \cdot G_{\xi}^{\mathrm{u}}
$$

which is almost semi-direct in the sense that $G_{\xi, \xi^{\prime}} \cap G_{\xi}^{\mathrm{u}}$ is compact. In particular, $G_{\xi}^{\mathrm{u}}$ acts transitively on $\mathrm{Opp}(\xi)$.

This algebraic Levi decomposition comes with the geometric counterpart below, which generalises the fact that, for semi-simple Lie groups, the Levi factor acts on a totally geodesic copy of its symmetric space embedded in the ambient space by the MostowKarpelevich theorem.

Proposition K. In the setting of Theorem $J$, the double stabiliser $G_{\xi, \xi^{\prime}}$ acts cocompactly on $P\left(\xi, \xi^{\prime}\right)$ and there is a canonical isometry $P\left(\xi, \xi^{\prime}\right) \cong \mathbf{R} \times X_{\xi}$ so that $G_{\xi, \xi^{\prime}} \cap \operatorname{Ker}\left(\beta_{\xi}\right)$ acts cocompactly on $X_{\xi}$.

(Herein $\beta_{\xi}: G_{\xi} \rightarrow \mathbf{R}$ denotes the Busemann character.)

As we shall see in Section 4 , the kernel of the $G_{\xi}$-action on $X_{\xi}$ is amenable. At this point, the following scheme emerges for a completely general cocompact group $G$ of isometries of a proper $\operatorname{CAT}(0)$ space $X$ :

If there is no global fixed point at infinity, then some structure results are provided in [CM09b]. Otherwise, we have a new cocompact $G$-action on a transverse space $X_{\xi}$. On the one hand, the kernel of this $G$-action is amenable, and thus described by Theorem A. On the other hand, the remaining quotient of $G$ is again a cocompact group of isometries, 
and we can therefore repeat this dichotomic analysis. This process terminates in finitely many steps since each transverse space will sit in $X$ with an additional Euclidean factor by Proposition K.

Another motivation for Theorem $\mathrm{J}$ is the study of $\mathrm{CAT}(0)$ lattices (in the sense of [CM09a]). Since many of the results in [CM09b], [CM09a] depend on the assumption that no point at infinity be fixed simultaneously by all isometries, it was quite valuable to know that uniform CAT(0) lattices (a.k.a CAT(0) groups) have no fixed point at infinity except possibly on the Euclidean factor (upon passing, if needed, to the canonical minimal invariant CAT(0) subspace). This was essentially established in [BS87], see [AB98, Cor. 2.7]. It has been generalised to finitely generated CAT(0) lattices in Proposition 3.15 of [CM09a]. However, none of these arguments seem to apply to infinitely generated lattices; but using Theorem $J$ we obtain the desired statement:

Theorem L. Let $X$ be a proper CAT(0) space without Euclidean factor and such that Is $(X)$ acts cocompactly and minimally.

If $\Gamma<\operatorname{Is}(X)$ is any lattice, then there are no $\Gamma$-fixed points at infinity.

As it turns out, the relevant consequence of Theorem J applies much more generally to unimodular groups:

Theorem M. Let $X$ be a proper $\mathrm{CAT}(0)$ space and $G<\mathrm{Is}(X)$ a closed subgroup acting cocompactly and minimally on $X$.

If $G$ is unimodular, then its fixed points at infinity are contained in the boundary of the Euclidean factor.

Location of the proofs. Theorems A, C and F, as well as their corollaries, are proved in the final section of the paper. They rely on the one hand, on some facts pertaining to the structure theory of general locally compact groups, which we collect in a preliminary Section 2, and on the other hand on specific geometric tools, which are developed in Sections 3 and 4 .

Section 3 discusses the transverse spaces and refined bordification of $X$. It culminates in a proof of the Levi decomposition (Theorem J and Proposition K). The amenability of the "unipotent radical" in the Levi decomposition is established in Section 4, using a notion of compactible subgroups that is analogous but quite more general than the compaction groups studied in [CCMT11]. Compactibility is some weak form of contractibility, which is confronted to the invariance of the Haar measure under conjugacy in order to deduce Theorems L and M. The final Section 5 is devoted to the proofs of the geometric and algebraic characterisations of amenable subgroups.

Acknowledgements. The final writing of this paper was partly accomplished when both authors were visiting the Mittag-Leffler Institute, whose hospitality was greatly appreciated. Thanks are also due to Ami Eisenmann for pointing out Corollary E.

\section{ON THE STRUCTURE OF LOCALLY COMPACT GROUPS}

It seems that all the problems concerning the structure of locally compact groups have been completely solved.

Hidehiko Yamabe, [Yam53, p. 352] 
According to the solution to Hilbert's fifth problem, which we shall refer to as Yamabe's theorem in the sequel, every locally compact group $G$ such that $G / G^{\circ}$ is compact has a unique maximal compact normal subgroup $W$ such that $G / W$ is a virtually connected Lie group (see [Yam53] and Theorem 4.6 in [MZ55]). This plays a fundamental role in the proof of the following.

Theorem 2.1. Let $Y$ be a locally compact group such that the group of components $Y / Y^{\circ}$ is locally elliptic.

Then $Y / \operatorname{Rad} \mathscr{L}_{\mathscr{E}}(Y)$ is a Lie group, $Y^{\circ} \operatorname{Rad} \mathscr{L}_{\mathscr{E}}(Y)$ is open in $Y$ and the discrete quotient $Y /\left(Y^{\circ} \operatorname{Rad}_{\mathscr{L} \mathscr{E}}(Y)\right)$ is virtually soluble.

The part of the statement not regarding virtual solubility was obtained in Theorem A.5 from [CT11].

We emphasize that the discrete quotient $Y /\left(Y^{\circ} \operatorname{Rad} \mathscr{L}_{\mathscr{E}}(Y)\right)$ need not be virtually torsion-free. Indeed, this is illustrated by the semi-direct product

$$
Y=\mathbf{R}^{2} \rtimes C_{p^{\infty}}<\mathbf{R}^{2} \rtimes O(2),
$$

where $C_{p}$ denote the group of all $p^{n}$-roots of unity with $p$ a prime and $n \geq 0$ an arbitrary integer. In this example the $\operatorname{radical} \operatorname{Rad}_{\mathscr{L} \mathscr{E}}(Y)$ is trivial and the group of components $Y / Y^{\circ} \cong C_{p^{\infty}}$ is abelian and locally elliptic, but not virtually torsion-free.

The proof of Theorem 2.1 requires some preparation and will be given at the end of this chapter.

2.A. Locally compact subgroups of Lie groups. Following the general convention in the theory of locally compact groups, we define a Lie group as a locally compact group $G$ such that the identity component $G^{\circ}$ is open in $G$ and is a connected Lie group in the usual sense.

Proposition 2.2. Let $G$ be a Lie group and $H$ be a locally compact group admitting a continuous faithful homomorphism into $G$.

Then we have the following.

(i) $H$ is a Lie group.

(ii) If $G / G^{\circ}$ is virtually soluble, then the following assertions are equivalent:

(a) $H / H^{\circ}$ is virtually soluble;

(b) $H / H^{\circ}$ is amenable;

(c) $H / H^{\circ}$ does not contain non-abelian free subgroups.

Proof. (i) is an immediate consequence of the characterization of Lie groups as those locally compact groups having no small subgroups, see [MZ55].

(ii) Assume that $G / G^{\circ}$ is virtually soluble. The implications (a) $\Rightarrow$ (b) $\Rightarrow$ (c) are clear. We assume henceforth that (c) holds. Let $\alpha: H \rightarrow G$ be a continuous faithful homomorphism, let $B=\overline{\alpha(H)}$ and $A=\overline{\alpha\left(H^{\circ}\right)}$. Thus $A$ is a closed connected normal subgroup of $B$, hence $A \leq B^{\circ}$.

By Theorem 2.1 from Chapter XVI in [Hoc65], the quotient of a connected Lie group by a dense connected normal subgroup is abelian. This implies that $A / \alpha\left(H^{\circ}\right)$ is abelian. So is thus $H^{1} / H^{\circ}$, where $H^{1}=\alpha^{-1}(A)$. Let also $H^{2}=\alpha^{-1}\left(B^{\circ}\right)$. Then the discrete group $H^{2} / H^{1}$ embeds in the connected Lie group $B^{\circ} / A$. By hypothesis $H^{2} / H^{1}$ does 
not contain non-abelian free subgroups. It must therefore be virtually soluble by the Tits alternative [Tit72]. Thus $H^{2} / H^{\circ}$ is virtually soluble, and it remains to show that $H / H^{2}$ is virtually soluble. Since the latter embeds continuously and faithfully in $B / B^{\circ}$, it suffices to prove that $B / B^{\circ}$ is virtually soluble. In other words, we have reduced the problem to the special case when $H=B$ is a closed subgroup of a Lie group $G$ with $G / G^{\circ}$ virtually soluble. This is what we assume henceforth.

Since $H^{\circ}$ is contained in $G^{\circ}$, there is no loss of generality in assuming that $G$ is connected.

Let $R$ be the soluble radical of $H^{\circ}$ and $S=H^{\circ} / R$. Let $J$ be the inverse image in $H$ of $\mathscr{Z}_{H / R}(S)$. Since $J$ is an extension of $R$ by a subgroup of $H / H^{\circ}$, it follows that $J$ does not contain non-abelian free subgroups. Moreover, since the outer automorphism group of the semisimple Lie group $S$ is finite, it follows that the image of $J$ in $H / H^{\circ}$ is of finite index. By the Tits alternative, a subgroup of a connected Lie group which does not have free subgroups must be virtually soluble. Thus $J$, and hence also $H / H^{\circ}$, is virtually soluble, as desired.

\section{B. Central extensions of locally elliptic groups.}

Proposition 2.3. Let $G$ be a locally compact group and $Z<G$ be a closed subgroup contained in the centre $\mathscr{Z}(G)$.

If $G / Z$ is locally elliptic, then $G / \operatorname{Rad} \mathscr{L}_{\mathscr{E}}(G)$ is abelian (and torsion-free).

Proposition 2.3 is a companion to the following classical result.

Theorem 2.4 (Ušakov [Uša63]). Let $G$ be a locally compact group in which every conjugacy class has compact closure.

Then the union of all compact subgroups of $G$ forms a closed normal subgroup, which therefore coincides with $\operatorname{Rad} \mathscr{L}_{\mathscr{E}}(G)$, and the corresponding quotient $G / \operatorname{Rad} \mathscr{L E}_{\mathscr{E}}(G)$ is abelian (and torsion-free).

Proof of Proposition 2.3. We are given a short exact sequence $1 \rightarrow Z \rightarrow G \rightarrow Q \rightarrow 1$ with $Z$ central in $G$ and $Q$ locally elliptic. Let $G^{\prime}=G / \operatorname{Rad} \mathscr{L}_{\mathscr{E}}(G)$ and $Z^{\prime}$ be the closure of the image of $Z$ in $G^{\prime}$; write $Q^{\prime}=G^{\prime} / Z^{\prime}$ and consider the short exact sequence

$$
1 \rightarrow Z^{\prime} \rightarrow G^{\prime} \rightarrow Q^{\prime} \rightarrow 1 .
$$

The normal subgroup $Z^{\prime}$ is central in $G^{\prime}$ (since the image of $Z$ is dense) and the quotient $Q^{\prime}$ is locally elliptic (since it is a quotient of $Q$ ). Thus the latter short exact sequence satisfies the hypotheses of the Proposition. We need to show that $G^{\prime}$ is abelian.

Let now $g_{1}, g_{2} \in G^{\prime}$. Then $\left\{g_{1}, g_{2}\right\}$ is contained in a group $H$ which an extension of $Z^{\prime}$ by a compact group since $Q^{\prime}$ is locally elliptic. Thus every conjugacy class in $H$ has compact closure, and it follows from Theorem 2.4 that $H / \operatorname{Rad}_{\mathscr{L} \mathscr{E}}(H)$ is abelian and torsion-free.

We first specialize this observation to the case when $g_{1}, g_{2}$ are both elliptic. It then follows that $g_{1}, g_{2} \in \operatorname{Rad}_{\mathscr{L} \mathscr{E}}(H)$, and thus that $g_{1}, g_{2}$ belong to a common compact subgroup of $G^{\prime}$. Therefore the set of elliptic elements of $G^{\prime}$ is a subgroup, and must therefore be contained in $\operatorname{Rad} \mathscr{L E}\left(G^{\prime}\right)=1$. It follows that $G^{\prime}$ has no nontrivial compact subgroup. 
Applying again the observation above to two arbitrary elements $g_{1}, g_{2}$, and taking now into account that $\operatorname{Rad}_{\mathscr{L} \mathscr{E}}(H)$ is trivial (since $G^{\prime}$ has no nontrivial compact subgroup), we infer that $H$ is abelian and, hence, that $g_{1}$ and $g_{2}$ commute. Thus $G^{\prime}$ is abelian, as desired.

2.C. Connected quotients and Proof of Theorem 2.1. The last tool that we need is the following fact; for the proof, we refer to Lemma 2.4 in [CCMT11].

Lemma 2.5. Let $G$ be a locally compact group with a quotient map $\pi: G \rightarrow L$ onto a Lie group L. Then $\pi\left(G^{\circ}\right)=L^{\circ}$.

As pointed out in [CCMT11], this statement can fail if $L$ is not Lie; for an elementary substitute in the general case, see Lemma 5.3 below.

Proof of Theorem 2.1. Let $Y$ be a locally compact group such that $Y / Y^{\circ}$ is locally elliptic. In order to prove the Theorem, we may assume without loss of generality that $\operatorname{Rad} \mathscr{L} \mathscr{E}\left(Y^{\circ}\right)=1$. Thus $Y^{\circ}$ is a connected Lie group by Yamabe's theorem. Theorem A.5 from [CT11] then yields that $Y^{\circ} \operatorname{Rad} \mathscr{L E}(Y)$ is open in $Y$. In particular $Y / \operatorname{Rad} \mathscr{L E}(Y)$ is a Lie group.

It remains to show that $Y /\left(Y^{\circ} \operatorname{Rad} \mathscr{L} \mathscr{E}(Y)\right)$ is virtually soluble.

Let $\pi: Y \rightarrow \operatorname{Aut}\left(Y^{\circ}\right)$ be the homomorphism induced by the conjugation action of $Y$ on $Y^{\circ}$. Since $\operatorname{Aut}\left(Y^{\circ}\right)$ acts faithfully on the Lie algebra of $Y^{\circ}$, we may assume that $\pi$ takes its values in $\mathrm{GL}_{n}(\mathbf{R})$ for some $n$. By Proposition 2.2, the group $\pi(Y)$ (endowed with the quotient topology from $Y / \operatorname{Ker}(\pi)$ ) is a Lie group. Consequently Lemma 2.5 yields $\pi\left(Y^{\circ}\right)=\pi(Y)^{\circ}$. In particular the group of components $\pi(Y) / \pi(Y)^{\circ}$ is a quotient of $Y / Y^{\circ}$, and is thus locally elliptic, hence amenable. Invoking Proposition 2.2 again, it follows that $\pi(Y) / \pi(Y)^{\circ}$ is virtually soluble.

Since $\operatorname{Ker}(\pi)=\mathscr{Z}_{Y}\left(Y^{\circ}\right)>\operatorname{Rad} \mathscr{L E}_{\mathscr{E}}(Y)$, all it remains to show is that

$$
Y^{\circ} \mathscr{Z}_{Y}\left(Y^{\circ}\right) / Y^{\circ} \operatorname{Rad} \mathscr{L}_{\mathscr{E}}(Y) \cong \mathscr{Z}_{Y}\left(Y^{\circ}\right) / Y^{\circ} \operatorname{Rad} \mathscr{L}_{\mathscr{E}}(Y) \cap \mathscr{Z}\left(Y^{\circ}\right)
$$

is virtually soluble. Observing further that

$$
Y^{\circ} \operatorname{Rad} \mathscr{L}_{\mathscr{E}}(Y) \cap \mathscr{Z}\left(Y^{\circ}\right)=\left(Y^{\circ} \cap \mathscr{Z}_{Y}\left(Y^{\circ}\right)\right) \operatorname{Rad} \mathscr{L}_{\mathscr{E}}(Y)=\mathscr{Z}\left(Y^{\circ}\right) \operatorname{Rad} \mathscr{L} \mathscr{E}(Y),
$$

the remaining statement to be proven is that $\mathscr{Z}_{Y}\left(Y^{\circ}\right) / \mathscr{Z}\left(Y^{\circ}\right) \operatorname{Rad} \mathscr{L}_{\mathscr{E}}(Y)$ is virtually soluble.

Notice that $Z=\mathscr{Z}\left(Y^{\circ}\right)$ is central in $\mathscr{Z}_{Y}\left(Y^{\circ}\right)$. Moreover the quotient $\mathscr{Z}_{Y}\left(Y^{\circ}\right) / Z \cong$ $Y^{\circ} \mathscr{Z}_{Y}\left(Y^{\circ}\right) / Y^{\circ}$ is locally elliptic, as it is isomorphic to a subgroup of $Y / Y^{\circ}$. We are thus in a position to apply Proposition 2.3 to the group $\mathscr{Z}_{Y}\left(Y^{\circ}\right)$. This implies that $\left.\mathscr{Z}_{Y}\left(Y^{\circ}\right) / \operatorname{Rad} \mathscr{L} \mathscr{E}_{(} \mathscr{Z}_{Y}\left(Y^{\circ}\right)\right)$ is abelian. Hence so are the groups $\mathscr{Z}_{Y}\left(Y^{\circ}\right) / \operatorname{Rad} \mathscr{L}_{\mathscr{E}}(Y)$ and $\mathscr{Z}_{Y}\left(Y^{\circ}\right) / \mathscr{Z}\left(Y^{\circ}\right) \operatorname{Rad} \mathscr{L} \mathscr{E}(Y)$. This concludes the proof.

\section{Horoactions And LeVi DeCOMpositions}

3.A. Transverse spaces and horoactions. To each point $\xi \in \partial X$ at infinity of a $\mathrm{CAT}(0)$ space $X$, one associates a new $\mathrm{CAT}(0)$ space $X_{\xi}$ called the transverse space of $\xi$ and defined as follows. Consider the set $X_{\xi}^{*}$ of all rays $r: \mathbf{R}_{+} \rightarrow X$ pointing to $\xi$. We claim that the infimal distance

$$
d_{\xi}\left(r, r^{\prime}\right)=\inf _{t, t^{\prime} \geq 0} d\left(r(t), r^{\prime}\left(t^{\prime}\right)\right)
$$


between rays is a pseudometric. Indeed, this follows from the convexity of the distance: more precisely, there is a constant $C$ (which is none other than the difference between the Busemann functions associated to $r$ and $r^{\prime}$ ) such that

$$
d_{\xi}\left(r, r^{\prime}\right)=\lim _{t \rightarrow \infty} d\left(r(t), r^{\prime}(t+C)\right)
$$

holds. Now we define $X_{\xi}$ to be the (Hausdorff) metric completion of the pseudometric space $X_{\xi}^{*}$, still denoting the resulting metric by $d_{\xi}$. The formula (3.i) shows that $X_{\xi}$ is a $\mathrm{CAT}(0)$ space and there is a canonical 1-Lipschitz map $X \rightarrow X_{\xi}$ with dense image.

Any isometry $g$ of $X$ induces an isometry $X_{\xi} \rightarrow X_{g \xi}$. In particular, there is a canonical isometric action of the stabiliser $\operatorname{Is}(X)_{\xi}$ on $X_{\xi}$.

Definition 3.1. The isometric $\operatorname{Is}(X)_{\xi}$-action on $\mathbf{R} \times X_{\xi}$ given by

$$
\begin{array}{rccc}
\omega_{\xi}: & G_{\xi} & \longrightarrow & \text { Is }\left(\mathbf{R} \times X_{\xi}\right) \\
\omega_{\xi}(g): & (t, x) & \longmapsto & \left(t+\beta_{\xi}(g), g \cdot x\right)
\end{array}
$$

is called the horoaction. Here, $\beta_{\xi}(g)$ is the Busemann character associated to $\xi$, which we recall can be expressed as

$$
\beta_{\xi}(g)=\lim _{t \rightarrow \infty}\left(d\left(r(t), x_{0}\right)-d\left(r(t), g x_{0}\right)\right),
$$

where $r$ is any ray pointing to $\xi$ and $x_{0}$ any point of $X$.

Remark 3.2. This definition shows that the kernel $\operatorname{Ker}\left(\omega_{\xi}\right)$ of the horoaction is precisely the normal subgroup $G_{\xi}^{\mathrm{u}} \triangleleft G_{\xi}$ introduced before the statement of Theorem J.

Some basic properties of the transverse space $X_{\xi}$ are collected in the following proposition. A point $\xi \in \partial X$ is called a cocompact point at infinity if its stabiliser $\operatorname{Is}(X)_{\xi}$ acts cocompactly on $X$.

Proposition 3.3. Let $\xi \in X_{\xi}$. We have the following.

(i) $X_{\xi}$ is a complete $\mathrm{CAT}(0)$ space.

(ii) The canonical homomorphism $\operatorname{Is}(X)_{\xi} \rightarrow \operatorname{Is}\left(X_{\xi}\right)$ is continuous.

(iii) If $X$ is of bounded geometry, then so is $X_{\xi}$; in particular, $X_{\xi}$ is proper in that case.

(iv) If $X$ is of bounded geometry, then it has finite depth.

(v) If a subgroup of $\operatorname{Is}(X)_{\xi}$ acts cocompactly on $X$, then it acts cocompactly on $X_{\xi}$. In particular, $X_{\xi}$ is a cocompact space whenever $\xi$ is a cocompact point.

Proof. For (i), (ii) and (iii), see Proposition 4.3 from [Cap09]. As for (iv), it follows from Corollary 4.4 in [Cap09]. The last item is straightforward.

Remark 3.4. Given a proper $\operatorname{CAT}(0)$ space $X$ and a closed subgroup $G \subseteq \operatorname{Is}(X)$ fixing a point $\xi \in \partial X$, we warn the reader that the image $\omega_{\xi}(G)$ of $G$ under the horoaction need not be closed in Is $\left(\mathbf{R} \times X_{\xi}\right)$. Indeed, consider the CAT(0) space $X=H \times T$, defined as the product of the hyperbolic plane $H=\mathbf{H}^{2}$ and the $p+1$-regular tree $T$, where $T$ is viewed as the Bruhat-Tits tree of $\mathrm{SL}_{2}\left(\mathbf{Q}_{p}\right)$. Let $G \subseteq \operatorname{Is}(X)$ be the infinite cyclic group $G=\left\{\left(\begin{array}{cc}1 & z \\ 0 & 1\end{array}\right) \mid z \in \mathbf{Z}\right\}$ diagonally embedded in $\mathrm{SL}_{2}(\mathbf{R}) \times \mathrm{SL}_{2}\left(\mathbf{Q}_{p}\right) \subseteq \operatorname{Is}(X)$. Then $G$ is discrete, hence closed in Is $(X)$. It fixes a point $\xi$ at infinity of the $\mathbf{H}^{2}$-factor, annihilates 
the Busemann character $\beta_{\xi}$ associated to that point, and fixes a point $x \in T$ in the tree factor.

Since $\xi$ lies on the boundary of the factor $H$, we have $X_{\xi}=(H \times T)_{\xi}=H_{\xi} \times T \cong T$ since $H_{\xi}$ is reduced to a singleton because $H$ is $\mathrm{CAT}(-1)$. In other words there is a canonical isometry $T \rightarrow X_{\xi}$ which is $\operatorname{Is}(X)_{\xi}$-equivariant. In particular the closure of the image $\omega_{\xi}(G)$ of $G$ under the horoaction in $\operatorname{Is}\left(\mathbf{R} \times X_{\xi}\right)$ is isomorphic to the closure of $G$ in $\mathrm{SL}_{2}\left(\mathbf{Q}_{p}\right)$, and is thus compact and infinite. In particular $\omega_{\xi}(G)$ is not closed.

\section{B. Lifting the transverse space.}

... quelque chose d'aussi naïf que l'entreprise d'atteindre l'horizon en marchant devant soi.

Marcel Proust, À la recherche du temps perdu - Albertine disparue,

Our next goal is to construct an isometric section $X_{\xi} \rightarrow X$ for the projection $X \rightarrow X_{\xi}$ when $\xi$ is a cocompact point at infinity (or opposite a cocompact point). We first record in Proposition 3.7 below an elementary observation which is valid under a much weaker assumption.

Definition 3.5. Let $G$ be any group acting by isometries on a CAT $(0)$ space $X$. We call $\xi \in \partial X$ a radial limit point (of $G$ ) if there is a sequence $\left\{g_{n}\right\}$ in $G$ such that for some ray $r_{0}: \mathbf{R}^{+} \rightarrow X$ pointing to $\xi$, the sequence $g_{n}^{-1} r_{0}(0)$ converges to $\xi$ while remaining at bounded distance of $r_{0}$.

Notice that in that case, the condition holds for all rays $r$ pointing to $\xi$. In fact, $g_{n}^{-1} x_{0}$ converges to $\xi$ while remaining at bounded distance of $r$ for any $x_{0} \in X$. Therefore we shall simply call such a sequence $\left\{g_{n}\right\}$ radial for $\xi$.

\section{Remarks 3.6.}

(1) If $X$ is cocompact, then any $\xi \in \partial X$ is a radial limit point.

(2) This definition should be distinguished from certain notions of conical limit points that are not equivalent in the absence of negative curvature (for instance, the terminology of [Hat05] is incompatible with that of [Alb99]).

(3) When no $G$ is given, it is always understood that $G=\operatorname{Is}(X)$.

For radial limit points, a simple limiting argument provides the following weak form of lifting, to be strengthened for cocompact points in Theorem 3.9 below.

Proposition 3.7. Let $X$ be a proper $\mathrm{CAT}(0)$ space. For every radial limit point $\xi \in \partial X$, there exists an isometric embedding $\mathbf{R} \times X_{\xi} \rightarrow X$.

The proposition has the following immediate consequence for the geometric dimension of the Tits boundary; a stronger statement for cocompact spaces will be given in Corollary 3.20 below.

Corollary 3.8. If $\xi \in \partial X$ is a radial point of the proper $\mathrm{CAT}(0)$ space $X$, then $\operatorname{dim}\left(\partial X_{\xi}\right) \leq \operatorname{dim}(\partial X)-1$.

Proof of Proposition 3.7. Let $r_{0},\left\{g_{n}\right\}$ be as in Definition 3.5. Let $t_{n} \geq 0$ be such that $r_{0}\left(t_{n}\right)$ is the projection of $g_{n}^{-1} r_{0}(0)$ to $r_{0}$. Then, for any given $r$ in $X_{\xi}^{*}$, the sequence 
$g_{n}\left(r\left(t_{n}\right)\right)$ remains bounded in $X$. Therefore there is a simultaneous accumulation point for the family (indexed by $r \in X_{\xi}^{*}$ ) of sequences of isometric maps

$$
\left[-t_{n}, \infty\right) \longrightarrow X, \quad t \longmapsto g_{n}\left(r\left(t+t_{n}\right)\right),
$$

uniformly on bounded intervals. This accumulation point is therefore a map $f^{*}$ from $X_{\xi}^{*}$ to the set of isometric maps $\mathbf{R} \rightarrow X$. By construction, any two maps in the image of $f^{*}$ remain at bounded distance. More precisely, the (Hausdorff) distance between $f^{*}(r)(\mathbf{R})$ and $f^{*}\left(r^{\prime}\right)(\mathbf{R})$ is exactly $d_{\xi}\left(r, r^{\prime}\right)$ for any $r, r^{\prime} \in X_{\xi}^{*}$. Denote by $T$ the metric space of all lines at finite distance of $f^{*}\left(r_{0}\right)(\mathbf{R})$ endowed with Hausdorff distance. The union of all these lines is a convex subset of $X$ isometric to the product $\mathbf{R} \times T$, see [BH99, II.2.14]; in fact this union is closed, i.e. $T$ is complete (compare e.g. the last sentence before Remark 40 in [Mon06]). The map $f^{*}: X_{\xi}^{*} \rightarrow T$ preserves (pseudo-)distances; therefore, $X_{\xi}$ is isometric to a subset of $T$, finishing the proof.

Following [CM09b], we say that a point $\xi^{\prime} \in \partial X$ is opposite to $\xi$ if there is a geodesic line in $X$ whose extremities are $\xi$ and $\xi^{\prime}$. We say that $\xi^{\prime}$ is antipodal to $\xi$ if $\angle_{\mathrm{T}}\left(\xi, \xi^{\prime}\right)=\pi$. Thus the set $\operatorname{Opp}(\xi)$ of points opposite to $\xi$ is contained in the set Ant $(\xi)$ consisting of all antipodes of $\xi$. It is shown in [CM09b, Proposition 7.1] that if $\operatorname{Is}(X)_{\xi}$ acts cocompactly, then $\operatorname{Opp}(\xi)$ is non-empty and any closed cocompact subgroup of $\operatorname{Is}(X)_{\xi}$ acts transitively on $\operatorname{Opp}(\xi)$.

Given $\xi^{\prime} \in \operatorname{Opp}(\xi)$, we denote by $P\left(\xi, \xi^{\prime}\right)$ the union of all geodesic lines joining $\xi$ to $\xi^{\prime}$. The set $P\left(\xi, \xi^{\prime}\right)$ is closed and convex in $X$; moreover there is a canonical isometric identification

$$
P\left(\xi, \xi^{\prime}\right) \cong \mathbf{R} \times Y
$$

for some complete CAT(0) space $Y$ (see [BH99, II.2.14]). When $\xi$ is cocompact, the following Theorem identifies $Y$ with $X_{\xi}$ and in particular provides an isometric section $X_{\xi} \rightarrow X$ for the canonical map $X \rightarrow X_{\xi}$.

Theorem 3.9. Assume that $\xi$ is cocompact.

Then, for any $\xi^{\prime} \in \mathrm{Opp}(\xi)$, the factor $Y$ in (3.ii) is canonicallly isometrically identified with $X_{\xi}$ under the map $X \rightarrow X_{\xi}$. Thus, there is a canonical isometric identification $P\left(\xi, \xi^{\prime}\right) \cong \mathbf{R} \times X_{\xi}$ which is $\operatorname{Is}(X)_{\xi, \xi^{\prime}}$-equivariant, the space $\mathbf{R} \times X_{\xi}$ being endowed with the horoaction.

Proof. Let $r_{0}: \mathbf{R} \rightarrow X$ be a geodesic line with $r_{0}(-\infty)=\xi^{\prime}$ and $r_{0}(+\infty)=\xi$. Since $\xi$ is cocompact, there is a sequence $\left\{g_{n}\right\}$ in $\operatorname{Is}(X)_{\xi}$ such that $d\left(g_{n} \cdot r_{0}(0), r_{0}(-n)\right)$ remains bounded. By convexity, $g_{n}\left(r_{0}(n)\right)$ remains bounded in $X$ and therefore we are in particular in the situation of the proof of Proposition 3.7. We thus obtain an isometric map

$$
\mathbf{R} \times X_{\xi} \longrightarrow P\left(\xi, \xi^{\prime}\right) \cong \mathbf{R} \times Y \subseteq X
$$

preserving the product decomposition, yielding in particular a (non-canonical) isometric embedding $\iota: X_{\xi} \rightarrow Y$. On the other hand, the restriction of $X \rightarrow X_{\xi}$ to $P\left(\xi, \xi^{\prime}\right)$ induces a canonical isometric map $\vartheta: Y \rightarrow X_{\xi}$ by the definition of $X_{\xi}$. Now $\vartheta \circ \iota$ is an isometric self-map of the space $X_{\xi}$ which is cocompact by Proposition 3.3(v). It follows that this self-map is onto (Proposition 4.5 in [CS11]) and thus $\vartheta$ is onto too. 
The action of $\operatorname{Is}(X)_{\xi, \xi^{\prime}}$ preserves the decomposition (3.ii) by construction and thus is a product action, see [BH99, I.5.3(4)]. The formula with $\beta_{\xi}(g)$ follows by direct computation.

The cocompactness of $\xi$ does not imply the cocompactness of its opposites (as illustrated by Heintze manifolds [Hei74]). Nevertheless, these opposites still enjoy the conclusions of Theorem 3.9. To make this statement precise, observe that the canonical isometry $P\left(\xi, \xi^{\prime}\right) \cong \mathbf{R} \times X_{\xi}$ induces a canonical isometric embedding $X_{\xi} \rightarrow X_{\xi^{\prime}}$.

Proposition 3.10. Let $\xi^{\prime}$ be any point opposite a cocompact point $\xi$. Then the canonical isometric embedding $X_{\xi} \rightarrow X_{\xi^{\prime}}$ is an isometry. In particular, $P\left(\xi, \xi^{\prime}\right) \cong \mathbf{R} \times X_{\xi^{\prime}}$ and this identification is compatible with the horoaction $\omega_{\xi^{\prime}}$ of $\operatorname{Is}(X)_{\xi, \xi^{\prime}}$.

Proof. Keep the notation of the proof of Theorem 3.9 and consider the ray $r_{0}^{-}: \mathbf{R}^{+} \rightarrow X$ defined by $r_{0}^{-}(t)=r_{0}(-t)$. Observe that the sequence $\left\{g_{n}^{-1}\right\}$ is as required in the proof of Proposition 3.7 with $\xi^{\prime}$ instead of $\xi$. We thus obtain an isometric embedding $\mathbf{R} \times X_{\xi^{\prime}} \rightarrow X$ such that one endpoint of the $\mathbf{R}$-factor is $\xi$; we denote the opposite endpoint by $\xi^{\prime \prime}$. We therefore have an isometric embedding of $\mathbf{R} \times X_{\xi^{\prime}} \rightarrow X$ into $P\left(\xi^{\prime \prime}, \xi\right) \cong \mathbf{R} \times Z$ (for some $Z$ ) preserving the product structures. Moreover, Theorem 3.9 provided canonical isometries

$$
P\left(\xi^{\prime}, \xi\right) \cong P\left(\xi^{\prime \prime}, \xi\right) \cong \mathbf{R} \times X_{\xi}
$$

preserving the product structures. At this point we have mutual isometric embeddings of $\mathbf{R} \times X_{\xi^{\prime}}$ and $\mathbf{R} \times X_{\xi}$ into each other and conclude as in the proof of Theorem 3.9 since $\mathbf{R} \times X_{\xi}$ is cocompact by Proposition 3.3(v).

Remark 3.11. It follows from Theorem 3.9 and Proposition 3.10 that if $\xi \in \partial X$ is a cocompact point, or if it is opposite a cocompact point, then the (non-Hausdorff) space $X_{\xi}^{*}$ is already complete and the map $X \rightarrow X_{\xi}$ is onto.

3.C. Existence of a 'Levi decomposition'. The following result will be our tool to investigate large stabilisers of points at infinity. Incidentally, it refines Proposition 7.1 in [CM09b] which stated that if $G_{\xi}$ acts cocompactly on $X$, then it acts transitively on $\operatorname{Opp}(\xi)$.

Theorem 3.12. Let $G<\operatorname{Is}(X)$ be a closed subgroup and assume $G_{\xi}$ acts cocompactly on $X$. Let $N<G_{\xi}$ be a subgroup that is normalised by some radial sequence $\left\{h_{n}\right\}$ in $G_{\xi}$ for $\xi$. Let $\xi^{\prime} \in \partial X$ be a limit point of $\left\{h_{n}\right\}$; hence $\xi^{\prime} \in \operatorname{Opp}(\xi)$.

Then, writing $N^{\mathrm{u}}=N \cap \operatorname{Ker}\left(\omega_{\xi}\right)$ where $\omega_{\xi}$ is the horoaction, we have a decomposition

$$
N=N_{\xi^{\prime}} \cdot N^{\mathrm{u}}
$$

which is almost semi-direct in the sense that $N_{\xi^{\prime}} \cap N^{\mathrm{u}}$ has compact closure. In particular $N^{\mathrm{u}}$ acts transitively on the $N$-orbit of $\xi^{\prime}$ in $\operatorname{Opp}(\xi)$.

The theorem applies in particular when $N$ is a normal subgroup of $G_{\xi}$. In that case, the above decomposition hold for any $\xi^{\prime} \in \mathrm{Opp}(\xi)$ since $G_{\xi}$ acts transitively on $\operatorname{Opp}(\xi)$ by Proposition 7.1 in [CM09b]. Thus we obtain Theorem $J$ as the case $N=G_{\xi}$, recalling Remark 3.2 for the notation $G_{\xi}^{\mathrm{u}}$.

We shall carry out the first part of the argument in a more general setting: for the time being, we only assume that $\xi$ is a radial limit point of $G$. 
Let $r_{0}$ be a ray pointing to $\xi$ and $\left\{g_{n}\right\}$ be any radial sequence as in Definition 3.5. At this point there is no additional restriction on $\left\{g_{n}\right\}$, though for Theorem 3.12 we will later choose $g_{n}=h_{n}$. As in the proof of Proposition 3.7, we have (upon passing to subsequences) a sequence of numbers $t_{n} \geq 0$ such that the sequence of maps

$$
\left[-t_{n}, \infty\right) \longrightarrow X, \quad t \longmapsto g_{n}\left(r_{0}\left(t+t_{n}\right)\right)
$$

converges to a geodesic line $\sigma: \mathbf{R} \rightarrow X$ uniformly on bounded intervals. In particular, $g_{n} \xi$ converges to the point $\eta:=\sigma(\infty)$ and $g_{n} r_{0}(0)$ to $\eta^{\prime}:=\sigma(-\infty)$. We denote by $f: \mathbf{R} \times X_{\xi} \rightarrow X$ the isometric embedding constructed in Proposition 3.7 and observe that $f$ ranges in $P\left(\eta, \eta^{\prime}\right)$ by construction.

A convexity argument shows that the sequence $\operatorname{Ad}\left(g_{n}\right)(g)=g_{n} g g_{n}^{-1}$ remains bounded in $G$ whenever $g \in G_{\xi}$. Therefore, we can chose a map $\varrho: G_{\xi} \rightarrow G$ that is a (point-wise) cluster point of the sequence $\left.\operatorname{Ad}\left(g_{n}\right)\right|_{G_{\xi}}$. By construction, $\varrho$ is a group homomorphism; it need not be continuous, see Example 3.14 below.

Proposition 3.13. In the above setting, the following hold.

(i) $\varrho\left(G_{\xi}\right) \subseteq G_{\eta, \eta^{\prime}}$,

(ii) $\operatorname{Ker}(\varrho) \subseteq \operatorname{Ker}\left(\omega_{\eta} \circ \varrho\right) \subseteq \operatorname{Ker}\left(\omega_{\xi}\right)$,

(iii) $\beta_{\eta} \circ \varrho=\beta_{\xi}$,

(iv) The isometric embedding $f: \mathbf{R} \times X_{\xi} \rightarrow P\left(\eta, \eta^{\prime}\right)$ is @-equivariant for $G_{\xi}$.

Proof. Points (i) and (iv) follow from the construction of $\varrho$ and $f$. Likewise for point (iii), using that the pointwise convergence on Busemann functions corresponds to the cone topology on the boundary.

Turning to (ii), let $g \in G_{\xi}$ be an element acting non-trivially on $X_{\xi}$. Then there is a ray $r_{1}$ asymptotic to $r_{0}$ and $\varepsilon>0$ such that $d\left(g r_{1}(t), r_{1}(s)\right) \geq \varepsilon$ for all $s, t \geq 0$. This implies that $\varrho(g)$ moves a line parallel to $\sigma(\mathbf{R})$ by at least $\varepsilon$. Thus $\omega_{\eta}(\varrho(g)) \neq 1$, which shows that $\operatorname{Ker}\left(\omega_{\eta} \circ \varrho\right)$ acts trivially on the transverse space $X_{\xi}$. Together with (iii), this establishes the non-trivial inclusion of (ii).

Proof of Theorem 3.12. We now assume that $G_{\xi}$ is cocompact and consider the radial sequence $\left\{h_{n}\right\}$ in $G_{\xi}$ given by hypothesis. We first observe that the image of $\left\{h_{n}\right\}$ in $\operatorname{Is}\left(X_{\xi}\right)$ is bounded and hence we can assume that it converges to some $\alpha \in \operatorname{Is}\left(X_{\xi}\right)$. We now specialise the above discussion to the case $g_{n}=h_{n}$. In particular, $\eta=\xi$ and $\eta^{\prime}=\xi^{\prime}$.

The sequence $\operatorname{Ad}\left(\omega_{\xi}\left(h_{n}\right)\right)$ converges to $\operatorname{Id} \times \operatorname{Ad}(\alpha)$ as automorphisms of $\operatorname{Is}\left(\mathbf{R} \times X_{\xi}\right)$ and hence the diagram

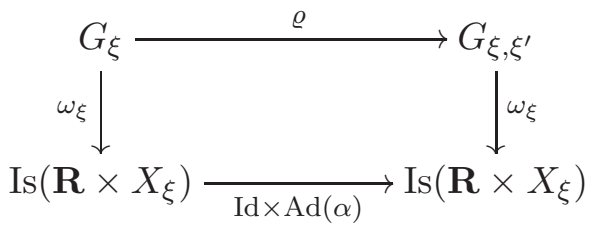

is commutative. Since each $h_{n}$ normalises $N$, the automorphism $\operatorname{Id} \times \operatorname{Ad}(\alpha)$ preserves $\omega_{\xi}(N)$. We deduce that $\omega_{\xi}(N)=\omega_{\xi}(\varrho(N)) \subseteq \omega_{\xi}\left(N_{\xi^{\prime}}\right)$ and therefore $\omega_{\xi}(N)=\omega_{\xi}\left(N_{\xi^{\prime}}\right)$. It follows that we have

$$
N=N_{\xi^{\prime}} \cdot \operatorname{Ker}\left(\left.\omega_{\xi}\right|_{N}\right)
$$

This provides the desired decomposition of $N$. 
For the compactness statement about $N_{\xi^{\prime}} \cap N^{\mathrm{u}}$, we claim that this intersection acts trivially on $P\left(\xi, \xi^{\prime}\right)$. Indeed, $N_{\xi^{\prime}}$ preserves $P\left(\xi, \xi^{\prime}\right)$ and the horoaction of $N^{\mathrm{u}}$ on $\mathbf{R} \times X_{\xi}$ is trivial; therefore, the claim follows from Theorem 3.9.

Proof of Proposition $K$. Pick $p_{0} \in P\left(\xi, \xi^{\prime}\right)$ and let $R>0$ be such that $X=G_{\xi} \cdot B\left(p_{0}, R\right)$. Given $p \in P\left(\xi, \xi^{\prime}\right)$, there is $g \in G_{\xi}$ such that $d\left(g . p, p_{0}\right)<R$. By Proposition 7.1 in [CM09b], $G_{\xi}$ acts transitively on $\operatorname{Opp}(\xi)$; therefore, the decomposition of Theorem 3.12 applied to $N=G_{\xi}$ shows that $G_{\xi}^{\mathrm{u}}=G \cap \operatorname{Ker}\left(\omega_{\xi}\right)$ also acts transitively. Thus there exists $k \in G_{\xi}^{\mathrm{u}}$ be such that $k g . \xi^{\prime}=\xi^{\prime}$; in particular we have $k g \in G_{\xi, \xi^{\prime}}$. Since both $p_{0}$ and $k g . p$ belong to $P\left(\xi, \xi^{\prime}\right)$, we deduce from Theorem 3.9 that

$$
\begin{aligned}
d\left(p_{0}, k g \cdot p\right)^{2} & =\left(b_{\xi}\left(p_{0}\right)-b_{\xi}(k g \cdot p)\right)^{2}+d\left(\pi_{\xi}(k g \cdot x), \pi_{\xi}\left(p_{0}\right)\right)^{2} \\
& =\left(b_{\xi}\left(p_{0}\right)-b_{\xi}(g \cdot p)\right)^{2}+d\left(\pi_{\xi}(g \cdot x), \pi_{\xi}\left(p_{0}\right)\right)^{2} \\
& \leq d\left(g \cdot x, p_{0}\right)^{2}+d\left(g \cdot x, p_{0}\right)^{2} \\
& <2 R^{2},
\end{aligned}
$$

where $\pi_{\xi}$ denotes the $G_{\xi}$ action on $X_{\xi}$. Thus we have $P\left(\xi, \xi^{\prime}\right) \subseteq G_{\xi, \xi^{\prime}} \cdot B\left(p_{0}, \sqrt{2} R\right)$, which confirms that $G_{\xi, \xi^{\prime}}$ acts cocompactly on $P\left(\xi, \xi^{\prime}\right)$. The additional statements now follow by applying Theorem 3.9.

The following example shows that $\varrho$ need not be continuous.

Example 3.14. Consider the simplicial line $L$ whose vertex set $\left(v_{n}\right)_{n \in \mathbf{Z}}$ is linearly ordered by the integers. Let $T$ be the tree obtained by adding to $L$ a collection of vertices of valency one, say $\left\{v_{n}^{\prime}, v_{n}^{\prime \prime}: n \in \mathbf{Z}\right\}$, where $v_{n}^{\prime}$ and $v_{n}^{\prime \prime}$ are declared adjacent to $v_{n}$. We view $T$ as a metric tree with all edges of length one. Thus $T$ is a CAT(0) space with two endpoints, say $\xi$ and $\xi^{\prime}$. The isometry group $G=\operatorname{Is}(T)$ acts cocompactly. Let $t \in G$ be the translation defined by $t:\left(v_{n}, v_{n}^{\prime}, v_{n}^{\prime \prime}\right) \mapsto\left(v_{n-1}, v_{n-1}^{\prime}, v_{n-1}^{\prime \prime}\right)$ for all $n$. We have $G_{\xi}=G_{\xi, \xi^{\prime}} \cong\left(\prod_{\mathbf{Z}} \mathbf{Z} / 2\right) \rtimes \mathbf{Z}$, where the cyclic factor $\mathbf{Z}$ is the group generated by $t$.

Let now $\varrho: G_{\xi} \rightarrow G$ be a limit point of the sequence $\left.\operatorname{Ad} t^{n}\right|_{G_{\xi}}$. We claim that $\varrho$ is not continuous.

Indeed, let $f_{n} \in G$ be defined by

$$
f_{n}:\left(v_{m}, v_{m}^{\prime}, v_{m}^{\prime \prime}\right) \mapsto\left\{\begin{array}{cl}
\left(v_{m}, v_{m}^{\prime}, v_{m}^{\prime \prime}\right) & \text { if } m<n \\
\left(v_{m}, v_{m}^{\prime \prime}, v_{m}^{\prime}\right) & \text { if } m \geq n
\end{array}\right.
$$

and set $f_{\infty}:\left(v_{m}, v_{m}^{\prime}, v_{m}^{\prime \prime}\right) \mapsto\left(v_{m}, v_{m}^{\prime \prime}, v_{m}^{\prime}\right)$ for all $m$. Then $\lim _{n} f_{n}=$ Id. On the other hand, for all $n$ and we have $\lim _{k} t^{k} f_{n} t^{-k}=f_{\infty}$ so that $\rho\left(f_{n}\right)=f_{\infty} \neq$ Id. In particular $\varrho$ is discontinuous at the identity.

Notice however that, although $\varrho$ is not continuous, the composite map $\omega_{\xi} \circ \varrho$ is always continuous, in view of the commutative diagram from the proof of Theorem 3.12, recalling that the horoaction is continuous by Proposition 3.3(ii).

3.D. Iterations and the refined bordification. The concept of transverse space suggests iterative constructions; this is formalised in the following definition.

Definition 3.15. A refining sequence for the CAT $(0)$ space $X$ is a sequence $\left(\xi_{1}, \ldots, \xi_{k}\right)$ with $k \geq 0, \xi_{1} \in \partial X$ and $\xi_{i+1} \in \partial X_{\xi_{1}, \ldots, \xi_{i}}$ for $i \geq 1$ ( $k=0$ corresponds to the empty sequence). The refined bordification of $X$ is the set of refined points $\left(\xi_{1}, \ldots, \xi_{k} ; x\right)$, wherein $\left(\xi_{1}, \ldots, \xi_{k}\right)$ is a refining sequence and $x \in X_{\xi_{1}, \ldots, \xi_{k}}$. Thus, $k=0$ corresponds 
simply to a point $x \in X$. We call $k$ the depth of the refined point. The depth of the space $X$ is the supremum of the depths of its refined points. (A characterisation of the depth of cocompact spaces will be given in Corollary 3.20 below.)

Thus the space $X$ is contained in the refined bordification, while $\partial X$ is a quotient of it.

Our next goal is to iterate the constructions described in the previous sections in order to lift transverse spaces of depth $>1$ in an equivariant way with respect to their stabilisers. Given a refining sequence $\left(\xi_{1}, \ldots, \xi_{k}\right)$, we endow the space

$$
\mathbf{R}^{k} \times X_{\xi_{1}, \ldots, \xi_{k}}
$$

with the refined horoaction (of depth $k$ ) of $\operatorname{Is}(X)_{\xi_{1}, \ldots, \xi_{k}}$, which is the product of the canonical action on $X_{\xi_{1}, \ldots, \xi_{k}}$ and the translation action on $\mathbf{R}^{k}$ provided by the product $\beta_{\xi_{1}} \times \cdots \times \beta_{\xi_{k}}$ of the Busemann characters.

The case $k=1$ in the following result is covered by Propositions 3.7 and 3.13.

Proposition 3.16. Let $X$ be a proper $\mathrm{CAT}(0)$ space and $G<\operatorname{Is}(X)$ be a closed subgroup acting cocompactly. For every refining sequence $\left(\xi_{1}, \ldots, \xi_{k}\right)$, there is an isometric embedding $f: \mathbf{R}^{k} \times X_{\xi_{1} \ldots, \xi_{k}} \rightarrow X$ and a (possibly discontinuous) homomorphism $\varrho: G_{\xi_{1}, \ldots, \xi_{k}} \rightarrow G$ such that $f$ is $\varrho$-equivariant for $G_{\xi_{1}, \ldots, \xi_{k}}$.

Proof. We shall construct inductively for $0 \leq i \leq k$ an isometric embedding

$$
f^{(i)}: \mathbf{R}^{i} \times X_{\xi_{1}, \ldots, \xi_{i}}
$$

and a homomorphism

$$
\varrho^{(i)}: G_{\xi_{1}, \ldots, \xi_{i}} \rightarrow G
$$

such that $f^{(i)}$ is $\varrho^{(i)}$-equivariant for $G_{\xi_{1}, \ldots, \xi_{i}}$. The final step $i=k$ will yield a map $f=f^{(k)}$ and a homomorphism $\varrho=\varrho^{(k)}$ enjoying the requested properties.

We start with $f^{(0)}=\mathrm{Id}: X \rightarrow X$ and $\varrho^{(0)}=\mathrm{Id}: G \rightarrow G$. For the inductive step, we now let $i>0$.

Viewing $X_{\xi_{1}, \ldots, \xi_{i-1}}$ as a subspace of $\mathbf{R}^{i-1} \times X_{\xi_{1}, \ldots, \xi_{i-1}}$, we may view $\xi_{i}$ as a point of $\partial\left(\mathbf{R}^{i-1} \times X_{\xi_{1}, \ldots, \xi_{i-1}}\right)$. It therefore makes sense to consider the image $f^{(i-1)}\left(\xi_{i}\right)$, which we denote by $\xi_{i}^{\prime} \in \partial X$. In particular we obtain an induced isometric embedding

$$
\mathbf{R}^{i-1} \times X_{\xi_{1}, \ldots, \xi_{i}} \rightarrow X_{\xi_{i}^{\prime}},
$$

which we also denote by $f^{(i-1)}$. The induction hypothesis ensures that the isometric embedding

$$
\mathrm{Id} \times f^{(i-1)}: \mathbf{R} \times \mathbf{R}^{i-1} \times X_{\xi_{1}, \ldots, \xi_{i}} \rightarrow \mathbf{R} \times X_{\xi_{i}^{\prime}}
$$

is $\varrho^{(i-1)}$-equivariant for $G_{\xi_{1}, \ldots, \xi_{i}}$ (where the domain $\mathbf{R} \times \mathbf{R}^{i-1} \times X_{\xi_{1}, \ldots, \xi_{i}}$ is endowed with the refined horoaction of depth $i$ of the stabiliser $G_{\xi_{1}, \ldots, \xi_{i}}$ ).

Since $G$ is cocompact, we can choose a sequence of isometries $\left\{g_{n}^{(i)}\right\}_{n}$ in $G$ that is radial for $\xi_{i}^{\prime}$. Propositions 3.7 and 3.13 then yield an isometric embedding

$$
\tilde{f}^{(i)}: \mathbf{R} \times X_{\xi_{i}^{\prime}} \rightarrow X
$$


and a homomorphism

$$
\tilde{\varrho}^{(i)}: G_{\xi_{i}^{\prime}} \rightarrow G
$$

such that $\tilde{f}^{(i)}$ is $\tilde{\varrho}^{(i)}$-equivariant for $G_{\xi_{i}^{\prime}}$. We then set

$$
f^{(i)}=\tilde{f}^{(i)} \circ\left(\operatorname{Id} \times f^{(i-1)}\right): \mathbf{R} \times \mathbf{R}^{i-1} \times X_{\xi_{1}, \ldots, \xi_{i}} \rightarrow X
$$

and

$$
\varrho^{(i)}=\tilde{\varrho}^{(i)} \circ \varrho^{(i-1)} .
$$

Since Id $\times f^{(i-1)}$ was shown to be $\varrho^{(i-1)}$-equivariant for $G_{\xi_{1}, \ldots, \xi_{i}}$, it follows that $f^{(i)}$ is $\varrho^{(i)}$-equivariant, which completes the induction step.

Remark 3.17. We record for later references that the homomorphism $\varrho$ is constructed as a composed map $\varrho=\tilde{\varrho}^{(k)} \circ \cdots \circ \tilde{\varrho}^{(1)}$, where each $\tilde{\varrho}^{(i)}$ is constructed as a pointwise limit, on its domain of definition, of a sequence of inner automorphisms of $G$.

The latter remark combines with the following elementary fact.

Lemma 3.18. Let $G$ be a second countable locally compact group, $H \subseteq G$ any subgroup and $\varrho_{1}, \ldots, \varrho_{k}$ a sequence of (possibly discontinuous) group homomorphisms with $\varrho_{1}: H \rightarrow G$ and $\varrho_{i+1}: \operatorname{Im}\left(\varrho_{i}\right) \rightarrow G$.

If each $\varrho_{i}$ is a pointwise limit, on its domain of definition, of a sequence of inner automorphisms of $G$, then so is the composed map $\varrho=\varrho_{k} \circ \cdots \circ \varrho_{1}: H \rightarrow G$.

Proof. It suffices to prove the statement for $k=2$; the general case follows by induction. Let thus $F$ be a finite subset of $H$ and $U$ be an open neighbourhood of $\varrho_{2}(H)$ in $G$. There is $\alpha \in \operatorname{Inn}(G)$ such that $\alpha\left(\varrho_{1}(F)\right) \subseteq U$. Since $\alpha$ is continuous, there is a neighbourhood $V$ of $\varrho_{1}(F)$ in $G$ such that $\alpha(V) \subseteq U$. Let $\beta \in \operatorname{Inn}(G)$ be such that $\beta(F) \subseteq V$. Then $\alpha \beta(F) \subseteq U$. Notice moreover that the inner automorphisms $\alpha$ and $\beta$ can be chosen in some fixed countable dense subgroup of $G$, which exists since $G$ is assumed second countable.

Repeating the argument for arbitrarily small neighbourhoods $U$ of $\varrho_{2}(H)$ and arbitrarily large finite subsets $F \subseteq H$, we get a net of inner automorphisms of $G$ of the form $\{\alpha \beta\}_{U, F}$, whose restrictions of $H$ have the composed homomorphism $\varrho=\varrho_{2} \circ \varrho_{1}$ as a limit point. By construction each element of the net belongs to a common countable subgroup of $G$; therefore the above net is actually a sequence.

Corollary 3.19. The homomorphism $\varrho: H \rightarrow G$ provided by Proposition 3.16 is a pointwise limit of a sequence of inner automorphisms of $G$.

Proof. Immediate from Remark 3.17 and Lemma 3.18, recalling that $G$ is second countable because $X$ is proper.

We warn the reader that, although $\varrho$ is a pointwise limit of a sequence $\left(\alpha_{n}\right)_{n \geq 0}$ of inner automorphisms of $G$, this does however not imply that $\left(\alpha_{n}\right)_{n>0}$ admits a subsequence whose restriction to $H$ converges pointwise to $\varrho$ (this reflects the fact that an uncountable product of metrizable topological spaces need not be first countable). However, there does exist such a subsequence in case the subgroup $H$ is countable.

The flat rank of a CAT(0) space is the supremum of the dimensions of its isometrically embedded Euclidean subspaces. By Theorem C in [Kle99], the flat rank of a cocompact 
proper $\operatorname{CAT}(0)$ space $X$ is given by $1+\operatorname{dim}(\partial X)$, where $\partial X$ is endowed with the Tits metric and dim is the supremum of the topological dimensions of compact subsets. Proposition 3.7 leads to an additional characterisation:

Corollary 3.20. Let $X$ be a cocompact proper CAT(0) space. Then the depth of $X$ coincides with its flat rank.

In fact, we have $\operatorname{dim}\left(\partial X_{\xi_{1}, \ldots, \xi_{k}}\right) \leq \operatorname{dim}(\partial X)-k$ for every refining sequence $\left(\xi_{1}, \ldots, \xi_{k}\right)$.

Proof. The embedding $\mathbf{R}^{k} \times X_{\xi_{1}, \ldots, \xi_{k}} \rightarrow X$ from Proposition 3.16 indeed yields the inequality $\operatorname{dim}\left(\partial X_{\xi_{1}, \ldots, \xi_{k}}\right) \leq \operatorname{dim}(\partial X)-k$. Taking a refined point of maximal depth, we deduce in particular that the flat rank of $X$ is at least its depth. On the other hand, it follows readily from the definitions that the flat rank of any CAT(0) space is a lower bound for its depth.

\section{Compactible subgroups and unimodularity}

4.A. Compactible subgroups. Let $G$ be a locally compact group. We propose the following generalization of the concept of contractible groups introduced by P. MüllerRömer [MR76].

A (not necessarily closed) subgroup $A<G$ is a compactible subgroup with limit $K$ if $K<G$ is a compact subgroup such that for every neighbourhood $U$ of $K$ in $G$ and every finite set $F \subseteq A$ there is $\alpha \in \operatorname{Aut}(G)$ such that $\alpha(F) \subseteq U$. (We write $\operatorname{Aut}(G)$ for the group of continuous automorphisms of $G$.)

A sequence $\left\{\alpha_{n}\right\}$ in $\operatorname{Aut}(G)$ such that $\alpha_{n}(a) \rightarrow K$ for all $a \in A$ will be called a compacting sequence, and we also call a sequence $\left\{g_{n}\right\}$ in $G$ compacting if the sequence of inner automorphisms $\operatorname{Ad}\left(g_{n}\right)$ is compacting.

The main difference with the notion of compaction groups from [CCMT11] is that we deform $A$ within the ambient group $G$ rather than only within itself.

Proposition 4.1. If $A<G$ is closed and compactible, then it is amenable.

Proof. By a result of Reiter's (Proposition 1 of $\S 3$ in [Rei71]), it suffices to prove the following: for every finite set $S \subseteq A$ there are non-negative $\varphi \in L^{1}(G)$ of integral one with $\|s \varphi-\varphi\|_{1}$ arbitrarily small for all $s \in S$.

Let thus $S$ be given. Let $\psi$ be some left $K$-invariant non-negative $\psi \in L^{1}(G)$ of integral one. By continuity of the $G$-representation on $L^{1}(G)$, there is a neighbourhood $V$ of the identity in $G$ such that $\|v \psi-\psi\|_{1}$ is as small as desired for all $v \in V$. By assumption there is $\alpha \in \operatorname{Aut}(G)$ such that $\alpha(S) \subseteq V K$. Let $\Delta_{G}(\alpha)$ be the modulus of $\alpha$ (VII $\S 1$ No 4 in [Bou04]); then $\varphi=\Delta_{G}(\alpha) \psi \circ \alpha$ has the required property.

The algebraic structure of a compactible subgroup can in fact be described more precisely using the following observation combined with Theorem 2.1.

Proposition 4.2. Let $G$ be a locally compact group and $A<G$ be a compactible subgroup. Let $Y=\overline{A \cdot G^{\circ}}$.

Then $Y / Y^{\circ}$ is locally elliptic.

Proof. Since $Y^{\circ}=G^{\circ}$, there is no loss of generality in assuming that $G$ is totally disconnected. 
Recall that every compact subgroup of a totally disconnected locally compact group is contained in a compact open subgroup (see Lemma 3.1 from [Cap09]). It follows that every finite set of elements in $A$ is contained in some compact subgroup of $G$. Thus $A$ is locally elliptic. Hence so is its closure $\bar{A}$ by Lemma 2.1 from [Cap09].

Corollary 4.3. Let $G$ be a locally compact group and $A<G$ be a closed compactible subgroup.

Then $A^{\circ} \operatorname{Rad}_{\mathscr{L} \mathscr{E}}(A)$ is open in $A$ and the discrete quotient $A /\left(A^{\circ} \operatorname{Rad} \mathscr{L} \mathscr{E}(A)\right)$ is virtually soluble.

Proof. Let $Y=\overline{A \cdot G^{\circ}}$. Thus $Y / Y^{\circ}$ is locally elliptic by Proposition 4.2. Let

$$
\pi: Y \rightarrow Y / \operatorname{Rad} \mathscr{L} \mathscr{E}(Y)
$$

be the canonical projection. By Theorem 2.1, the quotient $Y / \operatorname{Rad} \mathscr{L} \mathscr{E}(Y)$ is a Lie group whose group of components is virtually soluble. Moreover $A$, and hence also $A / A^{\circ}$, is amenable by Proposition 4.1. Therefore $\pi(A)$ is a Lie group and $\pi(A) / \pi(A)^{\circ}$ is virtually soluble by Proposition 2.2.

Since $A \cap \operatorname{Ker}(\pi)$ is contained in $\operatorname{Rad}_{\mathscr{L} \mathscr{E}}(A)$, we infer that $A / \operatorname{Rad}_{\mathscr{L} \mathscr{E}}(A)$ is a quotient of $\pi(A)$, and is thus itself a Lie group whose group of components is virtually soluble. From Lemma 2.5, it follows that $A^{\circ} \operatorname{Rad} \mathscr{L} \mathscr{E}(A) / \operatorname{Rad} \mathscr{L} \mathscr{E}(A)=(A / \operatorname{Rad} \mathscr{L} \mathscr{E}(A))^{\circ}$. Therefore $A / A^{\circ} \operatorname{Rad} \mathscr{L}_{\mathscr{E}}(A)$ is discrete and virtually soluble, as desired.

4.B. Compactible subgroups and the refined bordification. Given a refined point $\left(\xi_{1}, \ldots, \xi_{k} ; x\right)$, all the Busemann characters $\beta_{\xi_{i}}$ are well-defined on the iterated stabiliser $G_{\xi_{1}, \ldots, \xi_{k}}$ and hence also on $G_{\xi_{1}, \ldots, \xi_{k} ; x}$.

Proposition 4.4. Let $X$ be a proper $\mathrm{CAT}(0)$ space and $G<\operatorname{Is}(X)$ be a closed subgroup acting cocompactly. For any refined point $\left(\xi_{1}, \ldots, \xi_{k} ; x\right)$, there is a sequence in $G$ which is compacting for the stabiliser $G_{\xi_{1}, \ldots, \xi_{k} ; x} \cap\left(\bigcap_{i=1}^{k} \operatorname{Ker} \beta_{\xi_{i}}\right)$.

This strengthens a statement for totally disconnected groups contained in Proposition 4.5(iii) in [Cap09].

Proof. The group $G_{\xi_{1}, \ldots, \xi_{k} ; x} \cap\left(\bigcap_{i=1}^{k} \operatorname{Ker} \beta_{\xi_{i}}\right)$ is a point stabiliser for the refined horoaction of $G_{\xi_{1}, \ldots, \xi_{k}}$ on $\mathbf{R}^{k} \times X_{\xi_{1}, \ldots, \xi_{k}}$. Let $f: \mathbf{R}^{k} \times X_{\xi_{1}, \ldots, \xi_{k}} \rightarrow X$ and $\varrho: G_{\xi_{1}, \ldots, \xi_{k}} \rightarrow G$ be the maps provided by Proposition 3.16. The $\varrho$-equivariance of $f$ implies that $\varrho\left(G_{\xi_{1}, \ldots, \xi_{k} ; x}\right)$ fixes the point $f(x) \in X$, and is thus relatively compact in $G$. By Corollary 3.19, the homomorphism $\varrho$ is a pointwise limit of some sequence $\left(\operatorname{Ad} g_{n}\right)$ of inner automorphisms of $G$. Since $\varrho\left(G_{\xi_{1}, \ldots, \xi_{k} ; x}\right)$ is contained in the compact group $G_{f(x)}$, it follows that ( $\left.\operatorname{Ad} g_{n}\right)$ is a compacting sequence for $G_{\xi_{1}, \ldots, \xi_{k} ; x}$.

Corollary 4.5. Let $G$ be a locally compact group acting continuously, properly and cocompactly by isometries on $X$. Then the stabiliser of every point in the refined bordification is amenable.

Proof. Immediate from Propositions 4.1 and 4.4. 
4.C. Unimodular groups do not fix points at infinity. We begin with a general restriction for compactible subgroups in unimodular groups.

Proposition 4.6. Let $G$ be a unimodular locally compact group, $H<G$ a closed subgroup and $\left\{g_{n}\right\}_{n \in \mathbf{N}}$ a compacting sequence for $H$ in $G$.

If $\left\{g_{n} g g_{n}^{-1}\right\}$ remains bounded for each $g \in G$, then $H$ is compact.

Proof. Let $K<G$ be a compact subgroup with $g_{n} h g_{n}^{-1} \rightarrow K$ for all $h \in H$. Let further $U \subseteq G$ be any non-empty relatively compact open set. We claim that the union of all $g_{n} U g_{n}^{-1}$ is relatively compact.

For this claim there is no loss of generality in assuming $G$ to be $\sigma$-compact. For each compact set $C \subseteq G$, let $U_{C}=U \cap \bigcap_{n} g_{n}^{-1} C g_{n}$. As $C$ ranges over a countable exhaustion of $G$, the sets $U_{C}$ form a cover of $U$ by sets closed in $U$. Therefore, Baire's theorem implies that there is some compact $C$ such that $U_{C}$ has non-empty interior. Since $U$ has compact closure, we can find a finite set $F \subseteq G$ such that the set $U_{C} F$ covers $U$. Since the union of all $g_{n} F g_{n}^{-1}$ is relatively compact, the claim follows.

We thus have a compact set $Q \subseteq G$ containing all $g_{n} U g_{n}^{-1}$. Fix any compact neighbourhood $S$ of $K$ in $G$. Let $p$ be an integer such that $p|U|>|Q S|$, where $|\cdot|$ denotes a Haar measure on $G$. If, towards a contradiction, $H$ is non-compact, then we can find $h_{1}, \ldots, h_{p} \in H$ such that all $U h_{i}$ are disjoint. However, when $n$ is large enough, all $g_{n} U h_{i} g_{n}^{-1}$ lie in $Q S$, which is impossible by comparing the measures.

Proof of Theorem M. Let $\xi \in \partial X$ be a point fixed by the unimodular group $G$. By [CM09b, Prop. 7.1] the set $\operatorname{Opp}(\xi)$ is non-empty. Let $\xi^{\prime} \in \mathrm{Opp}(\xi)$. Theorem J yields a decomposition

$$
G=G_{\xi, \xi^{\prime}} \cdot G_{\xi}^{\mathrm{u}},
$$

where $G_{\xi}^{\mathrm{u}}=\operatorname{Ker}\left(\omega_{\xi}\right)$ is the kernel of the horoaction. Any radial sequence $\left\{g_{n}\right\}$ for $\xi$ is compacting for $G_{\xi}^{\mathrm{u}}$ (this is in fact a very basic case of Proposition 4.4). Moreover, $g_{n} g g_{n}^{-1}$ remains bounded for all $g$ in $G=G_{\xi}$, as observed in Section 3.C. Therefore, Proposition 4.6 implies that $G_{\xi}^{\mathrm{u}}$ is compact. However, a compact normal subgroup of a group acting minimally must be trivial. We deduce that $G=G_{\xi, \xi^{\prime}}$; in particular, $G$ preserves $P\left(\xi, \xi^{\prime}\right)$ which is thus equal to $X$. In conclusion, $X$ admits a product decomposition $X \cong \mathbf{R} \times X_{\xi}$ such that the pair $\left\{\xi, \xi^{\prime}\right\}$ corresponds to the boundary of the line-factor. In particular every point $\xi \in \partial X$ fixed by $G$ lies on the boundary of the Euclidean de Rham factor of $X$, as desired.

Proof of Theorem L. Since Is $(X)$ admits a lattice, it is unimodular. Therefore Theorem $M$ implies that $\operatorname{Is}(X)$ does not have a fixed point in $\partial X$. Now the "geometric Borel density" established in Theorem 1.1 of [CM09a] yields the conclusion.

\section{Amenable isometry groups}

La méthode de cet artificieux Bouillon, c'est [...] de rester, en fin de compte, le moyenneur des situations.

Charles-Augustin Sainte-Beuve, Causeries du lundi, tome 12, 1856 
5.A. Refined flats. We define a refined flat of depth $k$ in $X$ to be a flat contained in a space of the form $X_{\xi_{1}, \ldots, \xi_{k}}$, where $\left(\xi_{1}, \ldots, \xi_{k}\right)$ is a refining sequence. Thus a flat of depth 0 is a flat in $X$.

A key ingredient needed in the proof of Theorem A is the theorem by Adams-Ballmann from [AB98]. We shall use the following formulation of their result.

Proposition 5.1 (Adams-Ballmann). Let $X$ be a proper CAT(0) space of finite depth (e.g. cocompact, see Proposition 3.3(iv)).

Every amenable group acting continuously by isometries on $X$ stabilises a refined flat.

Proof. Follows from a repeated application of the Adams-Ballmann theorem [AB98]; the hypothesis that the depth is finite guarantees that the process terminates after finitely many steps.

Before proceeding to the proof of Theorem A, we shall undertake to prove Theorem F, providing a converse to Proposition 5.1 and thus establishing Corollary G. We point out that Theorem $\mathrm{F}$ is not a direct consequence of the amenability of the kernel of the refined horoaction, because the image of a (refined) flat stabiliser in the isometry group of that flat need not be closed, see Remark 3.4. Therefore, we first need to record a consequence of a theorem by Kazhdan-Margulis (see Theorem 8.16 from [Rag72]).

To this end, we recall that the space of all closed subgroups of a locally compact group $G$ is compact when endowed with the Chabauty topology [Cha50]. Moreover the Chabauty space of closed subgroups is metrisable provided $G$ is second countable; this happens e.g. when $G$ is a closed subgroup of $\operatorname{Is}(X)$, where $X$ is a proper metric space.

Proposition 5.2. Let $G$ be a locally compact group whose identity component $G^{\circ}$ has no non-trivial compact normal subgroup. Let $\left(\Gamma_{j}\right)_{j \in J}$ be a net of discrete free subgroups converging in the Chabauty topology to a closed subgroup $L \subseteq G$.

Then the neutral component $L^{\circ}$ is an abelian Lie group.

Proof. By van Dantzig's theorem [vD31, page 18], the group $G$ has an open subgroup $O$ (thus containing $G^{\circ}$ ) such that $O / G^{\circ}$ is compact. By Yamabe's theorem $\operatorname{Rad} \mathscr{L} \mathscr{E}(O)$ is compact and $G^{\circ}$ is a Lie group. Moreover Theorem 2.1 implies that $\operatorname{Rad} \mathscr{L E}(O) G^{\circ} \cong$ $\operatorname{Rad}_{\mathscr{L} \mathscr{E}}(O) \times G^{\circ}$ is open in $O$. Upon replacing $O$ by that subgroup, we shall assume henceforth that $O$ is a direct product of a connected Lie group and a compact (profinite) group.

Since $O$ is open, we have $L \cap O=\lim _{j}\left(\Gamma_{j} \cap O\right)$. Since the desired conclusion concerns the group $L^{\circ} \subseteq G^{\circ} \subseteq O$, there is therefore no loss of generality in assume that all the subgroups $\Gamma_{j}$ are contained in $O$ or, equivalently, that $G=O$. Then $G \cong K \times G^{\circ}$, where $K=\operatorname{Rad}_{\mathscr{L} \mathscr{E}}(G)$ is compact and the identity component $G^{\circ}$ is a Lie group. Since $G^{\circ}$ is a Lie group, so is $L^{\circ}$.

Let $\pi: G \rightarrow G^{\circ}$ be the canonical projection, which is proper since $K$ is compact. Thus $\pi\left(\Gamma_{j}\right)$ is a discrete free group for all $j$. Let $U$ be an open relatively compact identity neighbourhood of the connected Lie group $G^{\circ}$ as provided by the Kazhdan-Margulis theorem (see Theorem 8.16 from [Rag72]). Since $L^{\circ} \subseteq G^{\circ}$, we have

$$
L^{\circ}=\pi\left(L^{\circ}\right)=\left\langle U \cap \pi\left(L^{\circ}\right)\right\rangle \subseteq\langle U \cap \pi(L)\rangle \subseteq \lim _{j}\left\langle U \cap \pi\left(\Gamma_{j}\right)\right\rangle .
$$


The Kazhdan-Margulis Theorem ensures that $\left\langle U \cap \pi\left(\Gamma_{j}\right)\right\rangle$ is nilpotent, hence cyclic, for all $j$. Since a limit of abelian subgroups is abelian, it follows that $L^{\circ}$ is abelian.

We shall also need an elementary general fact related to Lemma 2.5 above:

Lemma 5.3. Let $N$ be a closed normal subgroup of a locally compact group $H$. Then the image of $H^{\circ}$ in $H / N$ is dense in $(H / N)^{\circ}$. In particular, if $N$ is compact, then we obtain an identification $H^{\circ} /\left(H^{\circ} \cap N\right) \cong(H / N)^{\circ}$.

This statement fails for general topological groups; recall for instance that $\mathbf{R}$ is the quotient of a totally disconnected group (see Exercice 17 for III $§ 2$ in [Bou60]).

Proof of the lemma. The image of $H^{\circ}$ in $H / N$ lies in $(H / N)^{\circ}$; denote its closure by $L$. Now $(H / N)^{\circ} / L$ is a connected group contained in $(H / N) / L$. Writing the latter as $H / \overline{H^{\circ} N}$, we see that it is a quotient of the totally disconnected group $H / H^{\circ}$. For locally compact groups, total disconnectedness passes to quotients (by van Dantzig's theorem [vD31, page 18]). Thus $(H / N)^{\circ} / L$ is also totally disconnected, hence trivial as claimed. The additional statement for $N$ compact follows from the properness of the map $H \rightarrow H / N$.

Here is a last preparation for the proof of Theorem F.

Lemma 5.4. Let $G$ be a group acting isometrically on a complete $\operatorname{CAT}(0)$ space $X$ and let $Y \subseteq X$ be a $G$-invariant closed convex subspace of finite coradius in $X$. If $G$ preserves a refined flat of $X$, then it also preserves a refined flat (of the same dimension) of $Y$.

Proof. The statement is formulated in such a way that it passes to transverse spaces, since $\partial X=\partial Y$ and since there is a canonical isometric map of finite coradius $Y_{\xi} \rightarrow X_{\xi}$ for all $\xi \in \partial X$. Therefore, it suffices to deal with the case of depth zero, i.e. a $G$-invariant flat $F \subseteq X$. Since the projection $\pi: X \rightarrow Y$ is $G$-equivariant, it is enough to show that $\pi$ is isometric on $F$. The function $x \mapsto d(x, Y)$ is convex and bounded, therefore it is constant on $F$. Denote this constant by $D$. We claim that $\pi(F)$ is convex; this claim will finish the proof because the function $y \mapsto d(y, F)$ is constant on $\pi(F)$ and therefore the sandwich lemma [BH99, II.2.12] will apply.

Let thus $x, x^{\prime} \in F$ and let $y$ be any point on the segment $\left[\pi(x), \pi\left(x^{\prime}\right)\right]$. By convexity of the metric, the distance between $y$ and the corresponding point $z$ on the segment $\left[x, x^{\prime}\right]$ (for simultaneous affine parametrisations) is at most $D$. However, $y$ is in $Y$ and $z$ is in $F$. By uniqueness of the projection, we deduce $y=\pi(z)$ and the claim is proved.

Proof of Theorem F. Suppose for a contradiction that $H<G:=\operatorname{Is}(X)$ is a nonamenable closed subgroup which stabilises a refined flat $F \subseteq X_{\xi_{1}, \ldots, \xi_{k}}$. Upon replacing $X$ by a minimal non-empty $G$-invariant closed convex subset, we may assume that $G$ acts minimally. Indeed, Lemma 5.4 ensures that $H$ still preserves a refined flat (still denoted by $F$ as above) and the restriction homomorphism to the isometries of the minimal subset has compact kernel. This reduction ensures that $G$ has no non-trivial compact normal subgroup, and hence the unique maximal compact normal subgroup $\operatorname{Rad} \operatorname{LE}_{\mathscr{E}}\left(G^{\circ}\right)$ of $G^{\circ}$ is trivial.

Notice that $H \cap\left(\bigcap_{i=1}^{k} \operatorname{Ker}\left(\beta_{\xi_{i}}\right)\right)$ is closed and co-amenable in $H$. Therefore it is nonamenable, and upon replacing $H$ by that intersection, we may assume that $H$ annihilates each Busemann character $\beta_{\xi_{i}}$. 
Let $N$ be the pointwise stabiliser of $F$ in $H$. Thus $N$ is amenable by Corollary 4.5, so that the quotient $H / N$ endowed with the quotient topology is non-amenable. The $H$-action on $F$ induces a continuous embedding $\pi: H / N \rightarrow \operatorname{Is}(F)$. Since $\operatorname{Is}(F)$ is a Lie group, so is $H / N$ by Proposition 2.2(i). Moreover, since $\operatorname{Is}(F)$ is amenable, the neutral component $(H / N)^{\circ}$ must be amenable as well, since non-amenable connected Lie groups cannot be continuously embedded in amenable Lie groups (this follows e.g. from the fact that any non-trivial continuous homomorphism of a simple Lie group is proper, see [BM96, Lemma 5.3] or [dC09]). Therefore the group of components $(H / N) /(H / N)^{\circ}$ is non-amenable, and hence contains a non-abelian free subgroup by Proposition 2.2(ii).

Denoting by $H^{1}$ the preimage of $(H / N)^{\circ}$ in $H$, we infer that $H^{1}$ is an open normal amenable subgroup of $H$, and that the quotient $H / H^{1}$ contains a non-abelian free subgroup. Let now $a, b \in H$ be elements which freely generate a non-abelian free subgroup modulo $H^{1}$. Then $\langle a, b\rangle \cap H^{1}$ is trivial, since otherwise there would be a word in the generators $a, b$ which gets killed in the quotient $H / H^{1}$, contradicting the presupposed freeness. It follows that $\Gamma=\langle a, b\rangle$ is a discrete non-abelian free subgroup of $H$, hence of $G$, which stabilises the refined flat $F \subseteq X_{\xi_{1}, \ldots, \xi_{k}}$, acts faithfully on $F$, and annihilates each Busemann character $\beta_{\xi_{i}}$.

Let $f: \mathbf{R}^{k} \times X_{\xi_{1}, \ldots, \xi_{k}} \rightarrow X$ and $\varrho: \Gamma \rightarrow G$ be the maps provided by Proposition 3.16. Thus $F^{\prime}=f\left(\mathbf{R}^{k} \times F\right)$ is a flat in $X$. Moreover, the equivariance afforded by Proposition 3.16 ensures that the map $\varrho$ is injective and that its image $\varrho(\Gamma)$ stabilises $F^{\prime}$.

By assumption the image of $\Gamma$ in the Lie group $\operatorname{Is}(F)$ is non-discrete. In particular the closure of its image has a non-trivial identity component. Consequently the closure of the image of $\varrho(\Gamma)$ in $\operatorname{Is}\left(F^{\prime}\right)$ has a non-trivial identity component.

Let $K$ be the pointwise stabiliser of $F^{\prime}$ in $G$. Thus $K$ is compact, and it follows from Lemma 5.3 that $\overline{\varrho(\Gamma)}^{\circ}$ has a non-trivial image in $\operatorname{Is}\left(F^{\prime}\right)$. Moreover Proposition 2.2(i) implies that $\overline{\varrho(\Gamma)}$ is open in $\overline{\varrho(\Gamma)}$.

By Corollary 3.19, the homomorphism $\varrho: \Gamma \rightarrow G$ is a pointwise limit of a sequence $\left(\alpha_{n}\right)_{n \geq 0}$ of inner automorphisms of $G$. Since $\Gamma$ is countable, we may assume, upon extracting, that the sequence of restrictions $\left(\left.\alpha_{n}\right|_{\Gamma}\right)_{n}$ converges pointwise to $\varrho$. Up to a further extraction, we may also assume that the sequence of conjugates $\alpha_{n}(\Gamma)$ converges in the Chabauty topology to some closed subgroup $L$. It then follows that $\varrho(\Gamma)$, and hence also the closure $\overline{\varrho(\Gamma)}$, is contained in $L$.

By Proposition 5.2 the identity component $L^{\circ}$ is an abelian Lie group. Thus $\overline{\varrho(\Gamma)}^{\circ}$ is an abelian open normal subgroup of $\overline{\varrho(\Gamma)}$. Therefore $\varrho(\Gamma) \cap \overline{\varrho(\Gamma)}^{\circ}$ is an abelian normal subgroup of $\varrho(\Gamma)$ which is dense in $\overline{\varrho(\Gamma)}^{\circ}$. By construction $\varrho(\Gamma)$ is a non-abelian free group, and has thus no non-trivial abelian normal subgroup. Consequently $\varrho(\Gamma) \cap \overline{\varrho(\Gamma)} \circ$ is trivial, and so is $\overline{\varrho(\Gamma)}^{\circ}$. This contradicts the fact that $\overline{\varrho(\Gamma)}^{\circ}$ has a non-trivial image in $\operatorname{Is}\left(F^{\prime}\right)$.

5.B. Structure of amenable closed subgroups. We finally record the following classical characterisation of amenability for connected locally compact groups:

Proposition 5.5. A connected locally compact group is amenable if and only if it is \{connected soluble\}-by-compact. 
Proof. Let $G$ be a connected locally compact group. By the solution of Hilbert's fifth problem [MZ55, 4.6], $G$ is compact-by-Lie. Furstenberg has shown that a connected Lie group is amenable if and only if it is \{connected soluble\}-by-compact [Fur63, Theorem 1.7]. Finally, Iwasawa has shown that the class of \{connected soluble\}-by-compact l.c. groups is closed under extensions [Iwa49, Theorem 18].

Proof of Theorem A. The 'if' part is clear. Conversely, let $H<\operatorname{Is}(X)$ be a closed amenable subgroup. Then $H^{\circ}$ is soluble-by-compact in view of Proposition 5.5 and hence (1) holds.

By Proposition 5.1, we find an $H$-invariant refined flat $F \subset X_{\xi_{1}, \ldots, \xi_{k}}$ of depth $k$. In particular we have a continuous homomorphism

$$
\varphi: H \rightarrow \operatorname{Is}(F) \cong \mathbf{R}^{n} \rtimes O(n),
$$

where $n=\operatorname{dim} F$. We endow the image $\varphi(H)$ with the quotient topology from $H$. By Proposition 2.2, the amenable locally compact group $\varphi(H)$ is a Lie group and its group of components $\varphi(H) / \varphi(H)^{\circ}$ is virtually soluble. By Lemma 2.5, we have $\varphi\left(H^{\circ}\right)=\varphi(H)^{\circ}$. Thus the normal subgroup $K=H^{\circ} \cdot \operatorname{Ker}(\varphi)$ is open in $H$ and the quotient $H / K \cong$ $\varphi(H) / \varphi(H)^{\circ}$ is virtually soluble.

The kernel $A=\operatorname{Ker}(\varphi)$ fixes $F$ pointwise, and is thus compactible by Proposition 4.4. Therefore $A^{\circ} \operatorname{Rad}_{\mathscr{L} \mathscr{E}}(A)$ is open in $A$ and the quotient $A / A^{\circ} \operatorname{Rad}_{\mathscr{L} \mathscr{E}}(A)$ is virtually soluble by Proposition 4.2. In particular the group

$$
H^{\circ} \cdot A^{\circ} \operatorname{Rad}_{\mathscr{L} \mathscr{E}}(A)=H^{\circ} \operatorname{Rad}_{\mathscr{L} \mathscr{E}}(A)
$$

is open in $K=H^{\circ} \cdot A$, hence in $H$. Since $\operatorname{Rad} \mathscr{L} \mathscr{E}(A)$ is characteristic in $A$, we have $\operatorname{Rad} \mathscr{L} \mathscr{E}(A) \leq \operatorname{Rad} \mathscr{L} \mathscr{E}(H)$, and we infer that $H^{\circ} \operatorname{Rad} \mathscr{L} \mathscr{E}(H)$ is open in $H$, which proves assertion (2).

For (3), recall from the above that $H / K$ and $A / A^{\circ} \operatorname{Rad} \mathscr{L} \mathscr{E}(A)$ are both virtually soluble. Therefore, so is $H^{\circ} A / H^{\circ} \operatorname{Rad} \mathscr{L} \mathscr{E}(A)=K / H^{\circ} \operatorname{Rad} \mathscr{L} \mathscr{E}(A)$. Virtual solubility being stable under group extensions, we infer that $H / H^{\circ} \operatorname{Rad} \mathscr{L} \mathscr{E}(A)$, and (3) follows since $\operatorname{Rad}_{\mathscr{L} \mathscr{E}}(A) \leq \operatorname{Rad}_{\mathscr{L} \mathscr{E}}(H)$.

The following lemma is well-known; a proof can be found in [dlHRV94, Proposition 4].

Lemma 5.6. A homomorphic image of a finitely generated group in any compact group is residually finite (thus the same holds for its image in a locally elliptic group).

Proof of Corollary B. Let $X$ be a cocompact proper CAT(0) space with an isometric $\Gamma$-action and let $H$ be the closure of the image of $\Gamma$ in $\operatorname{Is}(X)$. The image of $\Gamma$ in $H /\left(H^{\circ} \operatorname{Rad} \mathscr{L}_{\mathscr{E}}(H)\right)$ is trivial since this quotient is virtually soluble by Theorem A; thus $H=H^{\circ} \operatorname{Rad}_{\mathscr{L} \mathscr{E}}(H)$. Since $H^{\circ}$ is soluble-by-compact by Theorem A, we deduce from Lemma 5.6 that the image of $\Gamma$ in $H / \operatorname{Rad} \mathscr{L} \mathscr{E}(H)$ is soluble and hence trivial. It follows $H=\operatorname{Rad} \mathscr{L E}(H)$, but this implies that $H$ is compact since $\Gamma$ is finitely generated; we finally conclude that $H$ is trivial by applying again the residual finiteness argument.

We now turn to Corollary $\mathrm{H}$ and refer to [CFP96] for an introduction to Thompson's groups $F, T$ and $V$. Since $T$ and $V$ are simple and contain $F$, it suffices to produce a non-trivial kernel in $F$ to ensure that the entire $T$ - or $V$-action be trivial. Thus the following statement implies Corollary $\mathrm{H}$. 
Corollary 5.7. Any isometric action of Thompson's group $F$ on any proper cocompact $\mathrm{CAT}(0)$ space factors through the abelianisation $F \rightarrow F /[F, F] \cong \mathbf{Z}^{2}$.

Proof. Let $X$ be any proper cocompact CAT(0) space and assume that $F$ acts isometrically on $X$; let $\pi: F \rightarrow \operatorname{Is}(X)$ be the associated group homomorphism. According to Corollary 2.3 of [CM09a], the group $F$ fixes a point in the compactification $\overline{X^{\prime}}$ whenever it acts isometrically on any proper $\operatorname{CAT}(0)$ space $X^{\prime}$. Thus in particular it satisfies the conclusion of the Adams-Ballmann theorem and therefore we can deduce as in Proposition 5.1 that $F$ preserves a refined flat (in fact a refined point) for $X$. Now Theorem $\mathrm{F}$ implies that the closure $H<\operatorname{Is}(X)$ of $\pi(F)$ is amenable; we can therefore apply Theorem A. Recalling that the derived subgroup $[F, F]$ is simple, we deduce that $\pi([F, F])$ lies in $H^{\circ} \operatorname{Rad} \mathscr{L} \mathscr{E}(H)$. Recall next that $[F, F]$ contains many isomorphic copies of $F$; we chose one such subgroup $F_{1} \cong F$ in $[F, F]$. By Lemma 5.6, the homomorphism from $F_{1}$ to $\left(H^{\circ} \operatorname{Rad} \mathscr{L} \mathscr{E}(H)\right) / H^{\circ}$ must annihilate $\left[F_{1}, F_{1}\right]$ since the latter is simple. Thus $\pi\left(\left[F_{1}, F_{1}\right]\right)$ is contained in the soluble-by-compact group $H^{\circ}$. Choosing once again an isomorphic copy $F_{2} \cong F$ in $\left[F_{1}, F_{1}\right]$, one more application of Lemma 5.6 shows that $\pi\left(\left[F_{2}, F_{2}\right]\right)$ lies i $\mathrm{n}$ the soluble radical of $H^{\circ}$. This shows that $\pi\left(\left[F_{2}, F_{2}\right]\right)$ is trivial since $\left[F_{2}, F_{2}\right]$ is simple (non-abelian). We have established that $\pi$ has a non-trivial kernel; this implies that $\pi$ is trivial on all of $[F, F]$ since that group is simple.

The strategy of the above proof is the following: (i) prove a refined fixed point (or flat) property for a subgroup (not necessarily involving amenability); (ii) apply Theorem F to deduce that its closure in $\operatorname{Is}(X)$ is amenable; (iii) play off the structure provided by Theorem A against the structure of the given group.

This strategy can be implemented in many other cases; here is a first example:

Corollary 5.8. Let $G$ be a finitely generated group isomorphic to $G \times G$. For any isometric $G$-action on any proper cocompact $\mathrm{CAT}(0)$ space, there is a decomposition $G \cong G \times G$ for which that action factors through the first factor.

Moreover, if one chooses a priori decompositions $G \cong G^{n}$ for $n \in \mathbf{N}$, then for any isometric $G$-action on any proper cocompact $\mathrm{CAT}(0)$ space of flat rank $<n$, one of the $n$ factors in $G^{n}$ acts trivially.

We recall that every countable group can be embedded in a group $G \cong G \times G$ as above [Mei82, Cor. 6]. Moreover, there are torsion-free examples; for instance, following the proof of Proposition 7 in [Mei82], consider two copies $U, V$ of the Baumslag-Solitar group $B S(2,3)$ :

$$
U=\left\langle a, t: t^{-1} a^{2} t=a^{3}\right\rangle, \quad V=\left\langle b, s: s^{-1} b^{2} s=b^{3}\right\rangle .
$$

Then $t$ and $\left[a, t^{-1} a t\right]$ freely generate a free group, as do $\left[b, s^{-1} b s\right]$ and $s$. Define $T$ to be the free product of $U$ and $V$ amalgamated over these free subgroups (with the generators identified in the order given above). Then Meier exhibits a (non-trivial) finitely generated subgroup $G \cong G \times G$ in the full product $T^{\mathbf{N}}$. Notice that $T$ is obtained by successive HNN-extensions and amalgamated products, starting with infinite cyclic groups and amalgamating over free subgroups (of rank one and two). Elementary Bass-Serre theory (or classical HNN-theory [Neu54, §5]) thus implies that $T$ is torsion-free; hence $G$ is torsion-free as well. 
Proof of Corollary 5.8. It suffices to prove the second statement since the factors of $G^{n}$ can be suitably re-arranged as $G \times G$. Therefore, we fix some decomposition $G \cong G^{n}$ and an isometric $G$-action on a proper cocompact $\operatorname{CAT}(0)$ space $X$ of flat rank $<n$. In order to be able to pass to successive transverse spaces, we only retain the weaker information that $X$ is proper of flat rank $<n$ (which is preserved thanks to Proposition 3.16). Such a space cannot contain a product of $n$ unbounded convex subspaces. Therefore, the splitting theorem of [Mon06] (specifically, Corollary 10 therein) implies that either $G$ fixes a point at infinity of $X$, or one of the factors of $G^{n}$ preserves a bounded subset of $X$, hence fixing a point. In any case, it follows by induction that one factor $H \cong G$ of $G^{n}$ fixes a refined point. Applying successively Theorem $\mathrm{F}$ and Theorem A, we find that the image of $H$ is $\operatorname{Is}(X)$ is obtained by various extensions of soluble and residually finite groups (appealing to Lemma 5.6 for the residual finiteness). However, a finitely generated group isomorphic to its own square does not have any non-trivial finite or abelian quotient; thus $H$ acts trivially indeed.

It was pointed out in the introduction, as a consequence of Theorem $\mathrm{C}$, that the wreath product $\mathbf{Z} \imath \mathbf{Z}$ cannot be a discrete subgroup of $\operatorname{Is}(X)$ for any proper cocompact CAT(0) space $X$. The assumption of discreteness is essential in that observation, since the group $\mathbf{Z}$ \Z $\mathbf{Z}$ does admit faithful actions by automorphisms on regular locally finite trees. This should however be contrasted with the torison-free elementarily amenable group $G=((\mathbf{Z} \imath \mathbf{Z}) \prec \mathbf{Z}) \prec \mathbf{Z} \cdots$, which is more precisely defined as the increasing union of the groups

$$
G_{1}=\mathbf{Z}, \quad G_{n+1}=G_{n} \curlywedge \mathbf{Z}=\left(\bigoplus_{\mathbf{Z}} G_{n}\right) \rtimes \mathbf{Z},
$$

where $G_{n}$ is identified with its copy at the coordinate zero in $G_{n+1}$.

Corollary 5.9. For any isometric G-action on any proper cocompact CAT(0) space, there is a subgroup of $G$ isomorphic to $G$ which acts trivially.

Proof. This follows from Theorem A once one observes that the kernel of any homomorphism from $G$ to any soluble group and to any locally \{residually finite\} group contains a subgroup isomorphic to $G$.

5.C. Discrete amenable subgroups and soluble groups of finite rank. In view of Theorem A, discrete amenable subgroups of $\operatorname{Is}(X)$ are \{locally finite $\}$-by-\{virtually soluble\}. The main result of this section is the much more precise statement of Theorem C. We recall that a group is said be of Prüfer rank $r$ if every finitely generated subgroup can be generated by at most $r$ elements, and if $r$ is the smallest such integer. This notion is sometimes simply called the rank in the literature and we shall do so for brevity's sake. For example, the additive group $\mathbf{Q}^{n}$ is of rank $n$. Soluble groups of finite rank have been studied extensively since Mal'cev's foundational work in the early 1950's. An overview of their theory, including many far-reaching results can be consulted in the book [LR04]. At this point, we only record the elementary fact that the class of groups of finite rank is closed under group extensions: more precisely, if $G$ is a group with a normal subgroup $N$ such that $N$ and $G / N$ have rank $r$ and $r^{\prime}$ respectively, then $G$ has rank at most $r+r^{\prime}$. 
The proof of Theorem C has a structure roughly parallel to that of Theorem A, and will be given at the end of the section. We shall need to study stabilisers of refined flats; the discreteness assumption on $\Gamma$ is exploited in combination with some rigidity properties on discrete amenable subgroups of Lie groups.

Our first task is thus to collect those various subsidiary results, notably on Lie groups. Although they are probably well-known, we nevertheless supply proofs, by lack of appropriate references. The first is a variation on Bieberbach's theorem.

Proposition 5.10. Let $n \geq 0$. Given a closed subgroup $H$ of the Lie group $\mathbf{R}^{n} \rtimes O(n)$, the group of components $H / H^{\circ}$ is \{free abelian of rank $\left.\leq n\right\}$-by-finite.

Notice that the order of the finite quotient cannot be bounded by a function of $n$, since the circle group $S^{1}$ contains cyclic subgroups of arbitrarily large order.

Proof of Proposition 5.10. Let $\mathbf{E}^{n}$ be the Euclidean $n$-space, so that $\operatorname{Is}\left(\mathbf{E}^{n}\right)=\mathbf{R}^{n} \rtimes O(n)$. We work by induction on $n$, the base case $n=0$ being trivial.

If $H$ stabilises a proper Euclidean subspace, let $N$ be the (compact) kernel of the $H$ action on that space; thus $H / N$ can be identified with a closed subgroup of $\mathbf{R}^{m} \rtimes O(m)$ for $m<n$. By induction, $(H / N) /(H / N)^{\circ}$ is \{free abelian of rank $\left.\leq m\right\}$-by-finite. But this group is a quotient of $H / H^{\circ}$ by Lemma 5.3. The kernel of this quotient is finite, so that in this case we are done since a finite-by-\{free abelian of rank $\leq m\}$-by-finite group is $\{$ free abelian of rank $\leq m\}$-by-finite.

We assume henceforth that $H$ acts minimally on $\mathbf{E}^{n}$. Let $F \subset E_{n}$ be a minimal $H^{\circ}$-invariant Euclidean subspace, and set $m=\operatorname{dim}(F)$. The union of the collection of all such subspaces is itself a Euclidean subspace which is $H$-invariant, and must therefore coincide with $\mathbf{E}^{n}$ by minimality. Therefore we obtain an $H$-invariant product decomposition

$$
\mathbf{E}^{n} \cong \mathbf{E}^{m} \times \mathbf{E}^{n-m}
$$

with the additional property that the $H^{\circ}$-action on the second factor $\mathbf{E}^{n-m}$ is trivial. Let $p: H \rightarrow \operatorname{Is}\left(\mathbf{E}^{m}\right)$ and $p^{\prime}: H \rightarrow \operatorname{Is}\left(\mathbf{E}^{n-m}\right)$ be the canonical projections. We have seen that $H^{\circ} \leq \operatorname{Ker}\left(p^{\prime}\right)=H^{\prime}$.

Pick a base point $x \in \mathbf{E}^{m}$ and set $H_{x}=\operatorname{Stab}_{H}(x)$. Thus the intersection $H_{x} \cap H^{\prime}$ is compact, and hence the projection $p^{\prime}\left(H_{x}\right) \cong H_{x} /\left(H_{x} \cap H^{\prime}\right)$ is a closed subgroup of $\operatorname{Is}\left(\mathbf{E}^{n-m}\right)$.

By [DS02], the neutral component $H^{\circ}$ acts transitively on its minimal subspace $F \cong$ $\mathbf{E}^{m}$. Equivalently $p\left(H^{\circ}\right)$ is transitive on $\mathbf{E}^{m}$, and thus $H=H_{x} \cdot H^{\circ}$. In particular $H=H_{x} \cdot H^{\prime}$, hence we have $p^{\prime}(H) \cong H / H^{\prime} \cong H_{x} /\left(H_{x} \cap H^{\prime}\right) \cong p^{\prime}\left(H_{x}\right)$. Therefore, the projection $p^{\prime}(H)$ is a closed subgroup of $\operatorname{Is}\left(\mathbf{E}^{n-m}\right)$, which must be discrete since $p^{\prime}\left(H^{\circ}\right)$ is trivial. In particular $H / H^{\prime}$ is virtually \{free abelian of rank $\leq n-m$ \} by a version of Bieberbach's theorem, see e.g. Theorem 2 in [Olis0].

As $H^{\circ} \leq H^{\prime}$, the transitivity of $p\left(H^{\circ}\right)$ on $\mathbf{E}^{m}$ also implies $H^{\prime}=\left(H_{x} \cap H^{\prime}\right) \cdot H^{\circ}$. It follows that $H^{\prime} / H^{\circ}$ is compact, hence finite. Consequently $H / H^{\circ}$ is an extension of $H / H^{\prime}$ by a finite normal subgroup. Thus $H / H^{\circ}$ is virtually a free abelian group of rank $\leq n-m$, as desired.

Proposition 5.11. Let $G$ be a connected Lie group. Then there is $r=r(G)$ such that every discrete amenable subgroup $\Gamma<G$ has the following properties: 
(i) $\Gamma$ is $\{$ torsion-free polycyclic of rank $\leq r\}$-by-finite.

(ii) $\operatorname{Rad}_{\mathscr{L} \mathscr{E}}(\Gamma)$ is finite and $\Gamma / \operatorname{Rad}_{\mathscr{L} \mathscr{E}}(\Gamma)$ is $\{$ torsion-free polycyclic of rank $\leq r\}$-by$\{$ finite of order $\leq r\}$.

Proof. Let $\Gamma<G$ be a discrete amenable subgroup. Then $\Gamma$ is virtually soluble by Proposition 2.2. By Corollary 8.5 from [Rag72], any non-empty collection of soluble subgroups of $G$ has a maximal element. Recalling that polycyclic groups are precisely those soluble groups satisfying the ascending chain condition on subgroups (see e.g. [LR04, 1.3.1]), we infer that $\Gamma$ is virtually polycyclic. Consequently it is finitely generated and virtually \{torsion-free polycyclic\}. The bound on the rank of the polycylic kernel in terms of $G$ follows from [Aus60] (and can be traced back to Zassenhaus [Zas38]). Thus (i) holds, and the finiteness of $\operatorname{Rad}_{\mathscr{L} \mathscr{E}}(\Gamma)$ follows.

Let now $\Gamma<G$ be a discrete subgroup which is $\{$ torsion-free polycyclic of rank $n$ \}by-finite. In order to establish (ii), we proceed by induction on $n$, the case $n=0$ being trivial. Assume now $n>0$. Then $\Gamma$ has a non-trivial free abelian normal subgroup, say $\mathbf{Z}^{m} \cong A<\Gamma$, with $m \leq n$. The conjugation action of $\Gamma$ on $A$ yields a homomorphism $\pi: \Gamma \rightarrow \mathrm{GL}_{m}(\mathbf{Z})$ whose kernel is $\mathscr{Z}_{\Gamma}(A) \cdot A$.

The index of a torsion-free normal subgroup $\pi(\Gamma)$ is bounded above by a constant depending only on $m$. Therefore $\pi(\Gamma)$ is $\left\{\right.$ torsion-free polycyclic of rank $\left.\leq r^{\prime}\right\}$-by-\{finite of order $\left.\leq r^{\prime}\right\}$ for some $r^{\prime}$ depending only on $n$.

On the other hand, the induction hypothesis applies to the quotient $\operatorname{Ker}(\pi) / A$, which is a central extension of $\operatorname{Ker}(\pi)$. Bearing in mind that a central extension of a locally elliptic group is \{locally elliptic\}-by-\{torsion-free abelian\} (see Proposition 2.3), we infer that the group $\operatorname{Ker}(\pi) / \operatorname{Rad} \mathscr{L} \mathscr{E}(\operatorname{Ker}(\pi))$ is $\left\{\right.$ torsion-free polycyclic of rank $\left.\leq r^{\prime \prime}\right\}$-by\{finite of order $\left.\leq r^{\prime \prime}\right\}$ for some $r^{\prime \prime}$ depending only on $n$. Assertion (ii) follows since $\operatorname{Rad}_{\mathscr{L} \mathscr{E}}(\operatorname{Ker}(\pi))<\operatorname{Rad}_{\mathscr{L} \mathscr{E}}(\Gamma)$.

Finally, we record an abstract group theoretic property.

Lemma 5.12. Let $\Gamma$ be a countable group. Assume there are constants $r, M \geq 0$ such that for every finitely generated subgroup $\Lambda<\Gamma$, the radical $\operatorname{Rad}_{\mathscr{L} \mathscr{E}}(\Lambda)$ is finite and the quotient $\Lambda / \operatorname{Rad} \mathscr{L} \mathscr{E}(\Lambda)$ is $\{$ torsion-free polycyclic of rank $\leq r\}$-by-\{finite of order $\leq M\}$.

Then $\Gamma / \operatorname{Rad} \mathscr{L} \mathscr{E}(\Gamma)$ is $\{$ torsion-free soluble of rank $\leq r\}$-by-finite.

Proof. We enumerate the elements of $\Gamma$ as $\Gamma=\left\{\gamma_{1}, \gamma_{2}, \ldots\right\}$. For each $n>0$, let $\Lambda_{n}=$ $\left\langle\gamma_{1}, \ldots, \gamma_{n}\right\rangle$ and $R_{n}=\operatorname{Rad}_{\mathscr{L} \mathscr{E}}\left(\Lambda_{n}\right)$.

For $m \geq n$, the finite quotient $R_{n} / R_{m} \cap R_{n}$ embeds in $\Lambda_{m} / R_{m}$. Its order is thus bounded above by $M$. Since $R_{n}$ is finite, we find a sequence of indices $\left(m_{i}\right)_{i}$ such that $m_{i+1}>m_{i} \geq n$ and that the intersection $R_{m_{i}} \cap R_{n}$ is constant and has index at most $M$ in $R_{n}$. Setting $R_{n}^{\prime}=R_{m_{i}} \cap R_{n}$ and noticing that $\bigcap_{i} R_{m_{i}}$ is finite group that is normalised by all elements of $\Gamma$, we infer that $R_{n}^{\prime} \subseteq \bigcap_{i} R_{m_{i}} \subseteq \operatorname{Rad} \mathscr{L}_{\mathscr{E}}(\Gamma)$. This shows that $\left[R_{n}: R_{n} \cap \operatorname{Rad} \mathscr{L} \mathscr{E}(\Gamma)\right] \leq M$ for all $n$. It follows that the image of $\Lambda_{n}$ in the quotient $\Gamma / \operatorname{Rad} \mathscr{L} \mathscr{E}(\Gamma)$ is $\{$ finite of order $\leq M\}$-by-\{torsion-free polycyclic of rank $\leq r\}$-by-\{finite of order $\leq M\}$.

We infer that there is some $N$ such that every finitely generated subgroup of the quotient $H=\Gamma / \operatorname{Rad}_{\mathscr{L} \mathscr{E}}(\Gamma)$ is \{torsion-free polycyclic of rank $\left.\leq r\right\}$-by-\{finite of order $\leq$ $N\}$. In particular $H$ is of finite rank. It only remains to show that $H$ is virtually torsion-free. 
To this end, let $H^{\prime}$ be the subgroup of $H$ generated by all $N$ ! powers. Thus $H^{\prime}$ is normal and the quotient $H / H^{\prime}$ is of finite exponent $\leq N$ !. In particular, for any finitely generated subgroup $\Lambda<H$, the image of $\Lambda$ in $H / H^{\prime}$ is polycyclic-by-finite of finite exponent, hence finite. Consequently $H / H^{\prime}$ is locally finite. On the other hand $H / H^{\prime}$ is of finite rank since $H$ is so. Remark that a locally finite group of finite rank and of finite exponent must be finite by the solution to the restricted Burnside problem ${ }^{1}$ (see [Zel91] and references therein). We conclude that $H / H^{\prime}$ is finite.

Finally, given $h \in H^{\prime}$, there is a finitely generated subgroup $\Lambda$ of $H$ such that $h$ belongs to the subgroup $\Lambda^{\prime}$ of $\Lambda$ generated by all $N$ ! powers of elements of $\Lambda$. Since $\Lambda$ is $\{$ torsion-free $\}$-by- $\{$ finite of order $\leq N\}$, it follows that $\Lambda^{\prime}$ is torsion-free. Consequently $H^{\prime}$ is torsion-free as well.

Proof of Theorem $C$. Let $G=\operatorname{Is}(X)$ and $\Gamma<\operatorname{Is}(X)$ be a discrete amenable subgroup. We already know from Theorem $\mathrm{A}$ that $\Gamma / \operatorname{Rad}_{\mathscr{L}_{\mathscr{E}}}(\Gamma)$ is virtually soluble. We need to show that it is in fact virtually torsion-free soluble of rank $\leq r$ \} for some $r$ depending only on $X$.

We start with a preliminary observation.

Pick a basepoint $x \in X$ and let $R>0$ be large enough so that every $G$-orbit meets the closed ball $B(x, R)$ of radius $R$ around $x$. For each $z \in X$, the stabiliser $G_{z}$ is compact. Its image in the totally disconnected quotient $G / G^{\circ}$ is compact as well, and hence contained in some compact open subgroup of $G / G^{\circ}$ by [Cap09, Lemma 3.1]. Let $O_{z}<G$ be the preimage in $G$ of such a compact open subgroup. Thus $O_{z}$ is open, contains $G_{z} \cdot G^{\circ}$, and the quotient $O_{z} / G^{\circ}$ is compact.

We next remark that the union $\bigcup_{z \in B(x, R)} G_{z}$ is a relatively compact subset of $G$. We can therefore find a finite set $z_{1}, \ldots, z_{n}$ such that $\bigcup_{z \in B(x, R)} G_{z} \subset \bigcup_{i=1}^{n} O_{z_{i}}$. Let $O=\bigcap_{i=1}^{n} O_{z_{i}}$. Thus $O$ is open, hence contains $G^{\circ}$, and the quotient $O / G^{\circ}$ is compact.

We claim that there is a uniform bound $N$ such that for all $z \in B(x, R)$, we have $\left[G_{z} G^{\circ}: G_{z} G^{\circ} \cap O\right] \leq N$. The fact that this index is finite is clear since $O$ is open. Since $G_{z} G^{\circ} \subset \bigcup_{i=1}^{n} O_{z_{i}}$, it follows that $G_{z} G^{\circ}$ is covered by left cosets of $O$ in the various groups $O_{z_{i}}$. Therefore we obtain

$$
\left[G_{z} G^{\circ}: G_{z} G^{\circ} \cap O\right] \leq \sum_{i=1}^{n}\left[O_{z_{i}}: O\right] .
$$

The claim follows by setting $N=\sum_{i=1}^{n}\left[O_{z_{i}}: O\right]$.

We now return to the discrete amenable subgroup $\Gamma<G$. By Proposition 5.1, there is a $\Gamma$-invariant refined flat $F \subset X_{\xi_{1}, \ldots, \xi_{k}}$ of depth $k \geq 0$.

Let $f: \mathbf{R}^{k} \times X_{\xi_{1} \ldots, \xi_{k}} \rightarrow X$ and $\varrho^{\prime}: G_{\xi_{1}, \ldots, \xi_{k}} \rightarrow G$ be the maps provided by Proposition 3.16. By assumption $\Gamma$ stabilises the flat $\mathbf{R}^{k} \times F \subseteq \mathbf{R}^{k} \times X_{\xi_{1} \ldots, \xi_{k}}$. Since $f$ is $\varrho^{\prime}$-equivariant, it follows that $\varrho^{\prime}(\Gamma)$ stabilises the flat $F^{\prime}=f\left(\mathbf{R}^{k} \times F\right) \subseteq X$.

By cocompactness, there is some $h \in G$ such that $h\left(F^{\prime}\right)$ meets the ball $B(x, R)$. We set $\varrho=\operatorname{Ad} h \circ \varrho^{\prime}$, so that $\varrho(\Gamma)$ stabilises $h\left(F^{\prime}\right)$.

\footnotetext{
${ }^{1}$ It is quite possible that a softer argument could be provided in the present setting by invoking Corollary 2 page 141 of [Šun71].
} 
Let $H=\overline{\varrho(\Gamma)}$. Denote by $K<H$ the pointwise stabiliser of $h\left(F^{\prime}\right)$, which is thus compact and normal in $H$. The quotient $H / K$ embeds as a closed subgroup of the Lie group Is $\left(h\left(F^{\prime}\right)\right)$. Since $X$ is cocompact, the dimension of $F^{\prime}$ is bounded above by a constant depending only on $X$. By Lemma 2.5 (or Lemma 5.3), the neutral component $H^{\circ}$ maps onto the neutral component of $H / K$. Consequently Proposition 5.10 ensures that the quotient $H /\left(H^{\circ} K\right)$ is \{free abelian of rank $\leq r^{\prime}$ \}-by-finite, where $r^{\prime}=r^{\prime}(X)$ depends only on $X$. Let $\Gamma^{\prime}=\varrho^{-1}\left(H^{\circ} K\right)$. Thus $\Gamma / \Gamma^{\prime}$ is $\left\{\right.$ free abelian of rank $\left.\leq r^{\prime}\right\}$-byfinite.

By construction $K$ fixes some point $z \in B(x, R)$. Therefore we have $\varrho\left(\Gamma^{\prime}\right) \leq H^{\circ} K \leq$ $G^{\circ} G_{z}$. We finally set $\Gamma^{\prime \prime}=\varrho^{-1}(O)$. Thus $\left[\Gamma^{\prime}: \Gamma^{\prime \prime}\right] \leq\left[G^{\circ} G_{z}: G^{\circ} G_{z} \cap O\right] \leq N$ be the claim above.

Let $\Lambda<\Gamma^{\prime \prime}$ be a finitely generated subgroup. Thus $\varrho(\Lambda)<O$. Since $\Lambda$ is finitely generated and $O$ is open, it follows that the conjugate $\Lambda_{n}=h g_{n} \Lambda g_{n}^{-1} h^{-1}$ is contained in $O$ for all sufficiently large $n$.

By Theorem 2.1, the radical $\operatorname{Rad}_{\mathscr{L} \mathscr{E}}(O)$ is compact and the $\operatorname{group} O / \operatorname{Rad} \mathscr{L} \mathscr{E}(O)$ is an almost connected Lie group. Moreover, the number of its connected components depends only on $X$. Notice moreover that $\Lambda_{n} \cap \operatorname{Rad} \mathscr{L} \mathscr{E}(O)$ is a finite normal subgroup of $\Lambda_{n}$, thus contained in $\operatorname{Rad} \mathscr{L} \mathscr{E}\left(\Lambda_{n}\right)$. By applying Proposition 5.11 to the quotient $\Lambda_{n} / \Lambda_{n} \cap \operatorname{Rad} \mathscr{L} \mathscr{E}(O)$, we deduce that $\operatorname{Rad} \mathscr{L} \mathscr{E}\left(\Lambda_{n}\right)$ is finite and that $\Lambda_{n} / \operatorname{Rad} \mathscr{L} \mathscr{E}\left(\Lambda_{n}\right)$ is \{torsion-free polycyclic of rank $\left.\leq r^{\prime \prime}\right\}$-by-\{finite of order $\left.\leq r^{\prime \prime}\right\}$, where $r^{\prime \prime}$ depends only on $X$. The same properties therefore hold for $\Lambda$, since it is conjugate to $\Lambda_{n}$.

Recalling that $\left[\Gamma^{\prime}: \Gamma^{\prime \prime}\right] \leq N$, we infer that for every finitely generated subgroup $\Lambda \leq \Gamma^{\prime}$, the radical $\operatorname{Rad} \mathscr{L}_{\mathscr{E}}(\Lambda)$ is finitely generated and the quotient $\Lambda / \operatorname{Rad} \mathscr{L}_{\mathscr{E}}(\Lambda)$ is \{torsion-free polycyclic of rank $\left.\leq r^{\prime \prime}\right\}$-by- $\left\{\right.$ finite of order $\left.\leq N r^{\prime \prime}\right\}$. We are thus in a position to apply Lemma 5.12 to $\Gamma^{\prime}$, which ensures that $\Gamma^{\prime}$ is torsion-free soluble of rank $\left.\leq r^{\prime \prime}\right\}$-by-finite.

Recall further that $\Gamma / \Gamma^{\prime}$ is \{free abelian of rank $\leq r^{\prime}$ \}-by-finite. Therefore $\Gamma$ is \{torsion-free soluble of rank $\leq r\}$-by-finite, where $r=r^{\prime}+r^{\prime \prime}$ depends only on $X$. This concludes the proof.

5.D. An easy comment on nilpotent groups. For the record, we indicate a variation on the Adams-Ballmann theorem [AB98] that is apparently not available in the literature, giving a stronger conclusion in the special case of nilpotent groups. This easy comment is not needed for the results of this paper.

Proposition 5.13. Let $G$ be a nilpotent group acting by isometries on a proper $\operatorname{CAT}(0)$ space $X$. Then either the $G$ preserves a flat or it fixes a point $\xi \in \partial X$ and annihilates the Busemann character $\beta_{\xi}$.

This will be proved inductively, using the following relative statement for general groups.

Proposition 5.14. Let $G$ be a group acting by isometries on a proper CAT(0) space $X$. Then either the centre $\mathscr{Z}(G)$ preserves a flat or $G$ fixes a point $\xi \in \partial X$ and annihilates the Busemann character $\beta_{\xi}$.

Proof of Proposition 5.14. For any finite subset $F \subseteq \mathscr{Z}(G)$ and any $\epsilon>0$ we define

$$
X_{F, \epsilon}=\{x \in X: d(z x, x) \leq|z|+\epsilon \forall z \in F\},
$$


where $|z|$ denotes the infimal displacement length of $z$. By centrality, this closed convex subset of $X$ is non-empty and $G$-invariant. Letting $F$ grow and $\epsilon$ shrink, we obtain a net of subsets, monotone decreasing with respect to inclusion. Assume first that the intersection $Y$ of all $X_{F, \epsilon}$ is non-empty. Since all elements of $\mathscr{Z}(G)$ have constant displacement on $Y$, there is a decomposition $Y \cong \mathbf{R}^{n} \times Y_{0}$ (with $n \geq 0$ ) such that every element of $\mathscr{Z}(G)$ acts by (possibly trivial) translations on $\mathbf{R}^{n}$ and trivially on $Y_{0}$, see Theorem II.6.15 in [BH99]. Thus $\mathscr{Z}(G)$ preserves indeed a flat.

We assume henceforth that the net $X_{F, \epsilon}$ has empty intersection. This implies that the distance from a base-point $p$ to $X_{F, \epsilon}$ tends to infinity. Denote by $p_{F, \epsilon}$ the projection of $p$ to $X_{F, \epsilon}$. Any accumulation point $\xi \in \partial X$ of the net $p_{F, \epsilon}$ will correspond to a $G$ invariant Busemann function since $X_{F, \epsilon}$ is $G$-invariant, finishing the proof. (Compare Proposition 2.1(2) in [AB98].)

Proof of Proposition 5.13. We argue by induction on the nilpotency class of $G$; the base case is when $G$ is the trivial group. For the inductive step, we apply Proposition 5.14 and need only consider the case where $\mathscr{Z}(G)$ preserves a flat $F$ in $X$. Upon passing to a subflat of minimal dimension, we see that $F$ is minimal as (non-empty) convex $\mathscr{Z}(G)$ invariant subset. The union $U \subseteq X$ of all such flats splits canonically as $U \cong F \times C$ for some proper CAT $(0)$ space $C$ endowed with a canonical $G / \mathscr{Z}(G)$-action, see Theorem 4.3 in [CM09b]. We now apply the induction hypothesis to this $G / \mathscr{Z}(G)$-action on $C$. If $G / \mathscr{Z}(G)$ preserves a flat $E \subseteq C$, then $G$ preserves the flat $F \times E \subseteq X$ and we are done. If on the other hand $G / \mathscr{Z}(G)$ fixes a point $\xi \in \partial C \subseteq \partial X$ and annihilates the corresponding Busemann character, then we are also done since $\mathscr{Z}(G)$ acts trivially on $C$.

\section{REFERENCES}

[AB98] Scot Adams and Werner Ballmann, Amenable isometry groups of Hadamard spaces, Math. Ann. 312 (1998), no. 1, 183-195.

[Alb99] Paul Albuquerque, Patterson-Sullivan theory in higher rank symmetric spaces, Geom. Funct. Anal. 9 (1999), no. 1, 1-28.

[Aus60] Louis Auslander, Discrete solvable matrix groups, Proc. Amer. Math. Soc. 11 (1960), 687688.

[BH99] Martin R. Bridson and André Haefliger, Metric spaces of non-positive curvature, Grundlehren der Mathematischen Wissenschaften 319, Springer, Berlin, 1999.

[BM96] Marc Burger and Shahar Mozes, CAT(-1)-spaces, divergence groups and their commensurators, J. Amer. Math. Soc. 9 (1996), 57-93.

[Bou60] Nicolas Bourbaki, Éléments de mathématique. Première partie. (Fascicule III.) Livre III; Topologie générale. Chap. 3: Groupes topologiques. Chap. 4: Nombres réels, Troisième édition revue et augmentée, Actualités Sci. Indust., No. 1143. Hermann, Paris, 1960.

[Bou04] , Integration. II. Chapters 7-9, Elements of Mathematics (Berlin), Springer-Verlag, Berlin, 2004, Translated from the 1963 and 1969 French originals by Sterling K. Berberian.

[BS87] Marc Burger and Viktor Schroeder, Amenable groups and stabilizers of measures on the boundary of a Hadamard manifold, Math. Ann. 276 (1987), no. 3, 505-514.

[Cap09] Pierre-Emmanuel Caprace, Amenable groups and Hadamard spaces with a totally disconnected isometry group, Comment. Math. Helv. 84 (2009), 437-455.

[CCMT11] Yves Cornulier, Pierre-Emmanuel Caprace, Nicolas Monod, and Romain Tessera, Amenable hyperbolic groups, Preprint, 2011.

[CFP96] James W. Cannon, William J. Floyd, and Walter R. Parry, Introductory notes on Richard Thompson's groups, Enseign. Math. (2) 42 (1996), no. 3-4, 215-256. 
[Cha50] Claude Chabauty, Limite d'ensembles et géométrie des nombres, Bull. Soc. Math. France $\mathbf{7 8}$ (1950), 143-151.

[CM09a] Pierre-Emmanuel Caprace and Nicolas Monod, Isometry groups of non-positively curved spaces: discrete subgroups, J Topology 2 (2009), no. 4, 701-746.

[CM09b] Isometry groups of non-positively curved spaces: structure theory, J Topology 2 (2009), no. 4, 661-700.

[CS11] Pierre-Emmanuel Caprace and Michah Sageev, Rank rigidity for CAT(0) cube complexes, Geom. Funct. Anal. 21 (2011), no. 4, 851-891.

[CT11] Yves Cornulier and Romain Tessera, Contracting automorphisms and $L^{p}$-cohomology in degree one, Arkiv för Matematik 49 (2011), no. 2, 295-324.

[dC09] Yves de Cornulier, On lengths on semisimple groups, J. Topol. Anal. 1 (2009), no. 2, 113-121.

[dlHRV94] Pierre de la Harpe, A. Guyan Robertson, and Alain Valette, On exactness of group $C^{*}$ algebras, Quart. J. Math. Oxford Ser. (2) 45 (1994), no. 180, 499-513.

[DS02] Antonio J. Di Scala, Minimal homogeneous submanifolds in Euclidean spaces, Ann. Global Anal. Geom. 21 (2002), no. 1, 15-18.

[Far05] Daniel S. Farley, Actions of picture groups on CAT(0) cubical complexes, Geom. Dedicata 110 (2005), 221-242.

[Fur63] Harry Furstenberg, A Poisson formula for semi-simple Lie groups, Ann. of Math. (2) 77 (1963), 335-386.

[Hal74] P. Hall, On the embedding of a group in a join of given groups, J. Austral. Math. Soc. 17 (1974), 434-495, Collection of articles dedicated to the memory of Hanna Neumann, VIII.

[Hat05] Toshiaki Hattori, Geometric limit sets of higher rank lattices, Proc. London Math. Soc. (3) 90 (2005), no. 3, 689-710.

[Hei74] Ernst Heintze, On homogeneous manifolds of negative curvature, Math. Ann. 211 (1974), 23-34.

[Hoc65] G. Hochschild, The structure of Lie groups, Holden-Day Inc., San Francisco, 1965.

[Iwa49] Kenkichi Iwasawa, On some types of topological groups, Ann. of Math. (2) 50 (1949), 507-558.

[Kle99] Bruce Kleiner, The local structure of length spaces with curvature bounded above, Math. Z. 231 (1999), no. 3, 409-456.

[Lee00] Bernhard Leeb, A characterization of irreducible symmetric spaces and Euclidean buildings of higher rank by their asymptotic geometry, Bonner Mathematische Schriften, 326, Universität Bonn Mathematisches Institut, Bonn, 2000.

[LR04] John C. Lennox and Derek J. S. Robinson, The theory of infinite soluble groups, Oxford Mathematical Monographs, The Clarendon Press Oxford University Press, Oxford, 2004.

[Mei82] David Meier, Non-Hopfian groups, J. London Math. Soc. (2) 26 (1982), no. 2, 265-270.

[Mei85] _ Embeddings into simple free products, Proc. Amer. Math. Soc. 93 (1985), no. 3, 387-392.

[Mil68] John Milnor, Growth of finitely generated solvable groups, J. Differential Geometry 2 (1968), 447-449.

[Mon06] Nicolas Monod, Superrigidity for irreducible lattices and geometric splitting, J. Amer. Math. Soc. 19 (2006), no. 4, 781-814.

[MP12] Nicolas Monod and Pierre Py, An equivariant deformation of the hyperbolic space, Preprint, 2012.

[MR76] Peter R. Müller-Römer, Kontrahierende Erweiterungen und kontrahierbare Gruppen, J. Reine Angew. Math. 283/284 (1976), 238-264.

[MZ55] Deane Montgomery and Leo Zippin, Topological transformation groups, Interscience Publishers, New York-London, 1955.

[Neu54] Bernhard Hermann Neumann, An essay on free products of groups with amalgamations, Philos. Trans. Roy. Soc. London. Ser. A. 246 (1954), 503-554.

[Oli80] Richard K. Oliver, On Bieberbach's analysis of discrete Euclidean groups, Proc. Amer. Math. Soc. 80 (1980), no. 1, 15-21.

[Pla65] Vladimir P. Platonov, Lokal projective nilpotent radicals in topological groups., Dokl. Akad. Nauk BSSR 9 (1965), 573-577. 
[Rag72] Madabusi Santanam Raghunathan, Discrete subgroups of Lie groups, Springer-Verlag, New York, 1972, Ergebnisse der Mathematik und ihrer Grenzgebiete, Band 68.

[Rei71] Hans Reiter, $L^{1}$-algebras and Segal algebras, Springer-Verlag, Berlin, 1971, Lecture Notes in Mathematics, Vol. 231.

[Ros76] Shmuel Rosset, A property of groups of non-exponential growth, Proc. Amer. Math. Soc. 54 (1976), 24-26.

[Sch76] Paul E. Schupp, Embeddings into simple groups, J. London Math. Soc. (2) 13 (1976), no. 1, 90-94.

[Šun71] Vladimir Petrovich Šunkov, On locally finite groups of finite rank, Algebra and Logic 10 (1971), 127-142.

[Tit72] Jacques Tits, Free subgroups in linear groups, J. Algebra 20 (1972), 250-270.

[Uša63] V. I. Ušakov, Topological $\overline{F C}$-groups, Sibirsk. Mat. Ž. 4 (1963), 1162-1174.

[vD31] David van Dantzig, Studien over topologische algebra (proefschrift), Ph.D. thesis, Groningen, 1931.

[Weh73] Bertram A. F. Wehrfritz, Infinite linear groups. An account of the group-theoretic properties of infinite groups of matrices, Springer-Verlag, New York, 1973, Ergebnisse der Matematik und ihrer Grenzgebiete, Band 76.

[Yam53] Hidehiko Yamabe, A generalization of a theorem of Gleason, Ann. of Math. (2) 58 (1953), 351-365.

[Zas38] Hans Zassenhaus, Beweis eines Satzes über diskrete Gruppen., Abh. Math. Semin. Hansische Univ. 12 (1938), 289-312.

[Zel91] Efim I. Zelmanov, On the restricted Burnside problem, Proceedings of the International Congress of Mathematicians, Vol. I, II (Kyoto, 1990) (Tokyo), Math. Soc. Japan, 1991, pp. 395-402.

UCL - Math, Chemin du Cyclotron 2, 1348 Louvain-la-Neuve, Belgium

E-mail address: pe.caprace@uclouvain.be

EPFL, 1015 LAUSANne, Switzerland

E-mail address: nicolas.monod@epfl.ch 